EDITORIAL BOARD

ANTONIO CARCATERRA

ERIC A. CARLEN

FRANCESCO DELL'ISOLA

RAFFAELE ESPOSITO

ALBERT FANNJIANG

Gilles A. FranCFORT

PiERANGELO MARCATI

JEAN-JACQUES MARIGO

PETER A. MARKOWICH

MARTIN OSTOJA-STARZEWSKI

PIERRE SEPPECHER

DAVID J. STEIGMANN

PAUl STEINMANN

PierRe M. SuQueT

MANAGING EDITORS

MICOL AMAR

EMILIO BARCHIESI

ANGELA MADEO

MARTIN OSTOJA-STARZEWSKI

ADVISORY BOARD

ADNAN AKAY

Holm AltenbaCH

MICOL AMAR

HARM ASKES

TEODOR ATANACKOVIĆ

VICTOR BERDICHEVSKY

GUY BOUCHITTÉ

ANDREA BRAIDES

ROBERTO CAMASSA

MAURO CARFORE

ERIC DARVE

FELIX DARVE

ANNA DE MASI

Gianpietro Del Piero

EMMANUELE Di BENEDETTO

VICTOR A. EREMEYEV

BERNOLD FIEDLER

IRENE M. GAMBA

DAVID Y. GAO

Sergey GaVRILyuk

Timothy J. HEALEY

DOMINIQUe JEULiN

ROGER E. KHAYAT

Corrado LATTANZIO

ROBERT P. LIPTON

ANGELO LUONGO

ANGELA MADEO

JUAN J. MANFREDI

CARLO MARCHIORO ANIL MisRa

ROBERTO NATALINI PATRIZIO NEFF

Thomas J. PENCE

ANDREY PIATNITSKI

ERRICO PRESUTTI

MARIO Pulvirenti

LUCIO RUSSO

Miguel A. F. SAnjuaN

PATRICK SElVAdurai

MiroslaV ŠILHAVÝ

GUIDO SWEERS

ANTOINETTE TORDESILLAS

LEV TRUSKINOVSKY

JUAN J. L. VELÁZQUEZ

VINCENZO VESPRI

ANGELO VULPIANI msp.org/memocs

Università di Roma "La Sapienza", Italia

Rutgers University, USA

(CO-CHAIR) Università di Roma "La Sapienza", Italia

(TREASURER) Università dell'Aquila, Italia

University of California at Davis, USA

(CO-CHAIR) Université Paris-Nord, France

Università dell' Aquila, Italy

École Polytechnique, France

DAMTP Cambridge, UK, and University of Vienna, Austria

(CHAIR MANAGING EDITOR) Univ. of Illinois at Urbana-Champaign, USA

Université du Sud Toulon-Var, France

University of California at Berkeley, USA

Universität Erlangen-Nürnberg, Germany

LMA CNRS Marseille, France

Università di Roma "La Sapienza”, Italia

Università degli Studi dell'Aquila, Italy

Université de Lyon-INSA (Institut National des Sciences Appliquées), France

(CHAIR MANAGING EDITOR) Univ. of Illinois at Urbana-Champaign, USA

Carnegie Mellon University, USA, and Bilkent University, Turkey

Otto-von-Guericke-Universität Magdeburg, Germany

Università di Roma "La Sapienza", Italia

University of Sheffield, UK

University of Novi Sad, Serbia

Wayne State University, USA

Université du Sud Toulon-Var, France

Università di Roma Tor Vergata, Italia

University of North Carolina at Chapel Hill, USA

Università di Pavia, Italia

Stanford University, USA

Institut Polytechnique de Grenoble, France

Università dell'Aquila, Italia

Università di Ferrara and International Research Center MEMOCS, Italia

Vanderbilt University, USA

Gdansk University of Technology, Poland

Freie Universität Berlin, Germany

University of Texas at Austin, USA

Federation University and Australian National University, Australia

Université Aix-Marseille, France

Cornell University, USA

École des Mines, France

University of Western Ontario, Canada

Università dell' Aquila, Italy

Louisiana State University, USA

Università dell'Aquila, Italia

Université de Lyon-INSA (Institut National des Sciences Appliquées), France

University of Pittsburgh, USA

Università di Roma "La Sapienza", Italia

University of Kansas, USA

Istituto per le Applicazioni del Calcolo "M. Picone", Italy

Universität Duisburg-Essen, Germany

Michigan State University, USA

Narvik University College, Norway, Russia

Università di Roma Tor Vergata, Italy

Università di Roma "La Sapienza", Italia

Università di Roma "Tor Vergata", Italia

Universidad Rey Juan Carlos, Madrid, Spain

McGill University, Canada

Academy of Sciences of the Czech Republic

Universität zu Köln, Germany

University of Melbourne, Australia

École Polytechnique, France

Bonn University, Germany

Università di Firenze, Italia

Università di Roma La Sapienza, Italia

MEMOCS (ISSN 2325-3444 electronic, 2326-7186 printed) is a journal of the International Research Center for the Mathematics and Mechanics of Complex Systems at the Università dell'Aquila, Italy.

Cover image: "Tangle” by @ John Horigan; produced using the Context Free program (contextfreeart.org).

\section{PUBLISHED BY}

\section{I. mathematical sciences publishers} nonprofit scientific publishing http://msp.org/ 


\title{
EQUILIBRIUM PATHS OF HENCKY PANTOGRAPHIC BEAMS IN A THREE-POINT BENDING PROBLEM
}

\author{
EMILIO TURCO AND EMILIO BARCHIESI
}

\begin{abstract}
We investigate the mechanical behavior of so-called pantographic beams undergoing large deformations. To this aim, an exact-kinematics Hencky pantographic beam model has been employed in a three-point bending test. Given the occurrence of local snap-through instabilities and limit points, said Hencky model has been solved by means of a step-by-step strategy based on Riks's arclength method. Such a method has been particularly adapted for the case of problems with prescribed displacements, as opposed to those with prescribed forces. Numerical simulations performed by varying the stiffness parameters are discussed, aimed at getting an insight into the different behaviors which can be exhibited by pantographic beams. Numerical simulations performed by varying the quantity of unit cells for fixed total length allow instead to understand whether the observed features are inherent to the pantographic beam structure or size-dependent. Therefore, beyond being interesting for possible future engineering exploitation, we believe this phenomenological evidence to be useful in guiding the formulation of conjectures regarding observed microscale local snap-through instability phenomena in the framework of a previously proposed macroscale continuum model for pantographic beams obtained by asymptotic homogenization.
\end{abstract}

\section{Introduction}

In the recent literature, it has been widely argued, both experimentally and theoretically, that pantographic structures are very effective in reaching large total deformations while still operating in the elastic regime (see the review papers [dell'Isola et al. 2019a; 2019b] for a detailed introduction on pantographic structure properties and [dell'Isola et al. 2016] for an efficient and predictive model of such structures). Nevertheless, their properties at microscale induce further exotic properties at macroscale which can be exploited in several engineering applications. For instance, due to their characteristic accordion-like zero-energy mode, they may

\section{Communicated by Pierre Seppecher.}

Turco is the corresponding author.

MSC2010: 74B20.

Keywords: pantographic beams, Lagrangian models, nonlinear analysis. 
be used to build reconfigurable structures such as those reported in [Filipov et al. 2015]. A quantitative account of deformation mechanisms in unit elements of pantographic structures - including pantographic beams studied herein - has been given in [Turco et al. 2017a; 2019b], thus providing a way of assessing experimentally stiffnesses employed in Hencky modeling of such structures.

A kind of slender pantographic structure, the so-called pantographic beam, has been introduced in the literature as a microstructure leading by asymptotic homogenization to a complete second-gradient beam at macroscopic scale, which means a continuum beam model with elastic deformation energy depending on the projection of the second placement material gradient onto both the normal vector to the deformed centerline - i.e., the material curvature - and the tangent one i.e., the stretch gradient [Alibert et al. 2003; Barchiesi et al. 2018; 2019]. It has been demonstrated that, beyond the capacity of sustaining nonstandard boundary external actions like double-forces, unusual elastic nonlinear behaviors - like softening and apparent negative stiffness - can arise in the equilibrium path of such structures in, e.g., the three-point bending problem due to gradient behavior in extension.

In this work we focus on modeling by the Hencky approach [Turco et al. 2016c], with such pantographic beams subjected to a three-point bending test, and we aim to explore their nonlinear behaviors. As reported in the review paper [Bertoldi et al. 2017], there is a plethora of possible and fruitful applications which can take advantage of instability phenomena. In particular, one may conceive technical applications which exploit (i) the presence of buckling loads, e.g., to reach large reversible deformations triggered by loads fulfilling threshold criteria, (ii) multistability, i.e., the capability of snapping between two (or more) stable equilibrium configurations, or (iii) the possibility of introducing controlled defects to obtain multistability and, in general, to fine-tune the structural response so as to exhibit a desired behavior. If one considers that pantographic beams can be built at micrometer scale using the technology reported in [dell'Isola et al. 2019c], then all above points become actual and profitable possibilities.

Degenerate behaviors [Barchiesi et al. 2018; 2019] in the above-mentioned homogenized continuum model, i.e., local phase transition from flexible beam to cable, which can origin from local snap-through instabilities at microscale, for sufficiently large prescribed displacements, have been previously discussed. Approaching limit points of the equilibrium path - whose occurrence had been observed previously with discrete and continuum models - as well as when local snap-through instabilities occur, the analysis of the discrete model by means of numerical schemes based on the Newton-Raphson algorithm gets extremely ineffective. In such cases, e.g., Riks-like algorithms should be considered [Riks 1972]. In what follows we shortly introduce discrete model kinematics and deformation 
energy. We subsequently recall the employed Riks-like numerical method and socalled three-point bending problem for a discrete Hencky pantographic beam. We carry out a parametric study in order to explore the possible achievable nonlinear behaviors. It turns out as a novel result that the strongly nonlinear behavior of discrete pantographic beams is very rich and characterized by the occurrence of multiple limit points, by negative stiffness regions of the equilibrium path, and by the occurrence of local snap-through instability phenomena. These phenomena provide a strong microscale-based support to the formulation of conjectures regarding local beam-to-cable transitions in corresponding homogenized continua, which is one of the main motivations stimulating the present study.

\section{Lagrangian modeling}

We consider a straight pantographic beam in-plane such as that represented in Figure 1 (top). It consists of two families of extensional-bending bars connected in the intersecting midpoints by torsion links. Owing to previous experience in the realization and modeling of such links and said extensional-bending bars, we adopt the description depicted in Figure 1 (bottom) based on spring assemblies; see [Turco et al. 2016a; 2016b; 2016e; Turco and Rizzi 2016] for a description of the predictive capability of such a model, [Turco et al. 2017b] for a first attempt to optimize fiber orientation, and [Turco et al. 2018] for an enhanced Hencky model

$L$

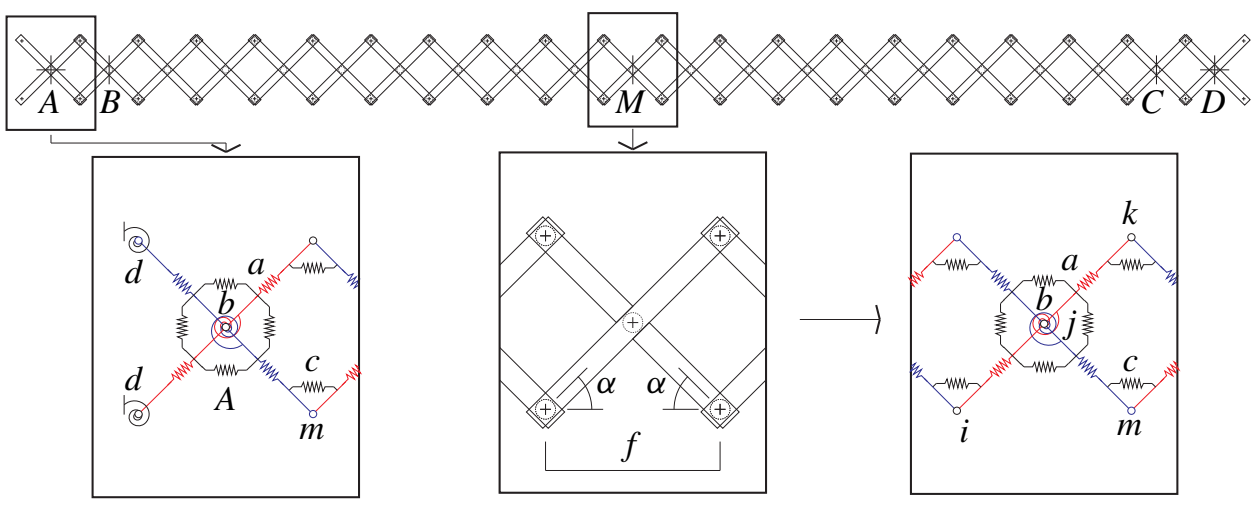

Figure 1. Geometry and Hencky model of considered pantographic beam (oblique bars form an angle $\alpha=\pi / 4$ with the horizontal line). In the three-point bending problem displacements of points $A$ and $D$, as well as vertical displacements of points $B$ and $C$, are set to be zero, while vertical displacement of the midpoint $M$ is fixed to a value different from zero. 
in pantographic structures with perfect pivots, i.e., pivots which behave as perfect hinges.

The finite-strain constitutive behaviors of elastically deformable links in the pantographic beam are the following ones, as expressed by means of stored deformation energies:

$$
\begin{aligned}
& E_{a}=\frac{1}{2} a \Delta \ell^{2}, \\
& E_{b}=b(1+\cos \beta), \\
& E_{c}=\frac{1}{2} c\left(\gamma-\frac{1}{2} \pi\right)^{2}, \\
& E_{d}=d\left(1-\cos \left(\delta-\frac{1}{4} \pi\right)\right),
\end{aligned}
$$

with $E_{a}, E_{b}, E_{c}$, and $E_{d}$ being the strain energies stored, respectively, in the extensional springs, depicted in red and blue in Figure 1, flexural-rotational springs, depicted in red and blue in Figure 1, shearing-rotational springs, depicted in black in Figure 1, and flexural-rotational springs on the left and right ends of the pantographic beam, depicted in black in Figure 1. The stiffnesses of the above-mentioned springs are denoted by $a, b, c$, and $d$, respectively ${ }^{1}$ the associated strain measures $\Delta \ell$ (stretching), $\cos \beta$ (bending), $\gamma$ (shearing), and $\delta$ (rotation) are all defined as functions of the current positions of nodes of the pantographic beam or, in other words, as functions of nodal displacements (nodes are depicted in Figure 1 as small circles located at the intersection points of the oblique bars) with respect to the reference configurations. Therefore, strain measures can be written as

$$
\begin{aligned}
\Delta \ell & =\left\|p_{j}-p_{i}\right\|-\left\|P_{j}-P_{i}\right\|, \\
\cos \beta & =\frac{\left\|p_{j}-p_{i}\right\|^{2}+\left\|p_{k}-p_{j}\right\|^{2}-\left\|p_{k}-p_{i}\right\|^{2}}{2\left\|p_{j}-p_{i}\right\|\left\|p_{k}-p_{j}\right\|}, \\
\gamma & =\arccos \frac{\left\|p_{j}-p_{m}\right\|^{2}+\left\|p_{k}-p_{j}\right\|^{2}-\left\|p_{k}-p_{m}\right\|^{2}}{2\left\|p_{j}-p_{m}\right\|\left\|p_{k}-p_{j}\right\|}, \\
\cos \left(\delta-\frac{1}{4} \pi\right) & =\frac{\boldsymbol{v} \cdot \boldsymbol{e}_{1}+\boldsymbol{v} \cdot \boldsymbol{e}_{2}}{\left\|P_{j}-P_{i}\right\|^{2}},
\end{aligned}
$$

where $P_{i}$ and $p_{i}$ are the reference and the current positions of node $i$ (the same is true for nodes $j, k$, and $m$ ), respectively, $\boldsymbol{v}=\left(p_{j}-p_{i}\right)-\left(P_{j}-P_{i}\right)$, and $\boldsymbol{e}_{1}, \boldsymbol{e}_{2}$ are the horizontal and vertical unit vectors (more details on this can be found in [Turco et al. 2016c]). Strain energies for each spring of the considered lattice completely define its mechanical behavior. We remark that the strain energy $E_{b}$ differs from

\footnotetext{
${ }^{1}$ Intentionally, although it would be possible, we do not assume as different the stiffnesses of the two families of oblique bars (red and blue rotational and extensional springs); at the same time we consider only one parameter $c$ to describe the shearing stiffness (black rotational springs) and one parameter $d$ to describe the flexural-rotational one on the left and right ends of the pantographic beam.
} 
that in [Barchiesi et al. 2018; 2019], which reads as $E_{b}=(b / 2)(\beta-\pi)^{2}$. It is easy to see that for $\beta$ in a small neighborhood of $\pi$ (i.e., the value assumed by $\beta$ in the reference zero-energy configuration) the two choices for $E_{b}$ are equivalent. Nevertheless, even for a relatively large neighborhoods - e.g., $\pi-1 \leq \beta \leq \pi+1$ (this neighborhood includes all values assumed by $\beta$ in the present analysis - it can be seen a posteriori) - the two energies behave so similarly as to give the same results for the scopes of the present analysis. This also shall motivate that for meaningfully relating discrete and continuum derivations, later on, we will use the same scaling law (see (14)) used in the continuum derivation [Barchiesi et al. 2018; 2019]. Defining the structural reaction of the system as the gradient of the total potential energy ${ }^{2}$ with respect to independent Lagrangian variables - e.g., nodal displacements - the stationarity condition for the potential energy with respect to independent Lagrangian variables, i.e., equating to zero the structural reaction, gives equilibrium equations. The Hessian matrix, which collects the second partial derivatives of the strain energy with respect to independent Lagrangian variables, is the so-called stiffness matrix of the system. The equilibrium equations and stiffness matrix of the considered system are the only tools which are necessary to code a step-by-step procedure such as that reported in the following section.

\section{Recovering the equilibrium path}

The nonlinear system of equilibrium equations derived by enforcing the stationarity condition for the potential energy with respect to nodal displacements reads as

$$
s[\boldsymbol{u}, \lambda \overline{\boldsymbol{u}}]=\mathbf{0},
$$

with $\boldsymbol{s}$ being the structural reaction depending upon vectors $\boldsymbol{u}$ and $\lambda \overline{\boldsymbol{u}}$. The vector $\boldsymbol{u}$ collects nodal displacements relative to the nodes which are free to be displaced, while the vector $\overline{\boldsymbol{u}}$ collects those nodal constrained displacements scaled by the real parameter $\lambda$. We remark that this kind of problem, i.e., a problem with constrained displacements on a subset of nodal displacements, is not usual in technical literature with few exceptions [Turco 2018b; Turco et al. 2019a]. Therefore, we report below a brief description of the used method and the final formulae. We further explicitly remark that $\lambda$, still being the parameter which scales the nodal constrained displacements to give $\bar{u}$, is also the value of the only nonzero constrained (vertical) displacement, i.e., that at the middle of the beam (node $M$ ).

The solution of the nonlinear system of equations (3) in the unknown nodal displacements can be achieved by using a step-by-step procedure based on Newton's method. If the pair $\left(\boldsymbol{u}_{i}, \lambda_{i}\right)$ - where index $i$ is not referring to node numbering

${ }^{2}$ Potential and strain energy are the same in the considered case, since the work of external loads is zero. 
as in (2) but to iterations of a recursive procedure - represents an equilibrium point, that is $s\left[\boldsymbol{u}_{i}, \lambda_{i} \overline{\boldsymbol{u}}\right]=\mathbf{0}$, then - notwithstanding that there may be multiple equilibrium points for fixed $\Delta \lambda$ - we can estimate a further close equilibrium point $\left(\boldsymbol{u}_{i}+\Delta \boldsymbol{u}, \lambda_{i}+\Delta \lambda\right)$ by linearizing (3):

$$
s\left[\boldsymbol{u}_{i}, \lambda_{i} \overline{\boldsymbol{u}}\right]+\boldsymbol{K}_{u u} \Delta \boldsymbol{u}+\boldsymbol{K}_{u \bar{u}} \Delta \lambda \overline{\boldsymbol{u}} \approx \mathbf{0},
$$

where $\boldsymbol{K}_{\boldsymbol{u} \boldsymbol{u}}$ and $\boldsymbol{K}_{\boldsymbol{u} \overline{\boldsymbol{u}}}$ are submatrices of the stiffness matrix $\boldsymbol{K}$, computed in $\left(\boldsymbol{u}_{i}, \lambda_{i}\right)$, whose rows correspond to the variables collected in the vector $\boldsymbol{u}$ and whose columns correspond to the variables collected in the vectors $\boldsymbol{u}$ and $\overline{\boldsymbol{u}}$, respectively. The same notation will be used to indicate submatrices of the stiffness matrix in the sequel.

We can then estimate for a chosen $\Delta \lambda$ - which should be small enough so that the point fulfills up to a given residual tolerance the equilibrium equations unknown displacements as the sum of $\boldsymbol{u}_{i}$ and the increment of displacements $\Delta \boldsymbol{u}$ found as

$$
\Delta \boldsymbol{u}=-\boldsymbol{K}_{u \boldsymbol{u}}^{-1} \boldsymbol{K}_{u \bar{u}} \Delta \lambda \overline{\boldsymbol{u}}=\Delta \lambda \hat{\boldsymbol{u}},
$$

which is nothing but Newton's method. An iteration scheme based on (5) can be simply built by updating the stiffness matrix at each step, i.e., pure Newton's method, or using the same stiffness matrix for multiple iterations (i.e., modified Newton's method). It is well known that Newton's method fails when $\boldsymbol{K}_{\boldsymbol{u} u}$ is singular or nearly singular, e.g., in or close to limit points of the equilibrium path. In order to overcome this limitation, Riks [1972] proposed to reconstruct the equilibrium path by parametrizing it by its arc length instead of by the parameter $\lambda$. This produces a scheme which does not suffer from the convergence problems of Newton's method, while requiring an additional constraint equation. In this scheme we aim to find an equilibrium point $\left(\boldsymbol{u}_{i}+\Delta \boldsymbol{u}+\dot{\boldsymbol{u}}, \lambda_{i}+\Delta \lambda+\dot{\lambda}\right)$ by using as said additional constraint equation a kind of orthogonality ${ }^{3}$ condition between the extrapolation $(\Delta \boldsymbol{u}, \Delta \lambda)$, given by the Newton's method (see (5)), and Riks's correction $(\dot{\boldsymbol{u}}, \dot{\lambda})$. Starting from a known equilibrium point $\left(\boldsymbol{u}_{i}, \lambda_{i}\right)$, the correction $(\dot{\boldsymbol{u}}, \dot{\lambda})$ to the Newton extrapolation $\left(\boldsymbol{u}_{i}+\Delta \boldsymbol{u}, \lambda_{i}+\Delta \lambda\right)$ can be computed by exploiting the linearization

$$
\boldsymbol{s}\left[\boldsymbol{u}_{i}+\Delta \boldsymbol{u},\left(\lambda_{i}+\Delta \lambda\right) \overline{\boldsymbol{u}}\right]+\boldsymbol{K}_{u \boldsymbol{u}} \dot{\boldsymbol{u}}+\boldsymbol{K}_{\boldsymbol{u} \overline{\boldsymbol{u}}} \dot{\lambda} \overline{\boldsymbol{u}} \approx \mathbf{0},
$$

where $\boldsymbol{K}_{\boldsymbol{u} \boldsymbol{u}}$ and $\boldsymbol{K}_{\boldsymbol{u} \overline{\boldsymbol{u}}}$ have been defined in the foregoing, but are now computed in $\left(\boldsymbol{u}_{i}+\Delta \boldsymbol{u}, \lambda_{i}+\Delta \lambda\right)$. From (6) we can evaluate $\dot{\boldsymbol{u}}$ as

$$
\dot{\boldsymbol{u}}=-\boldsymbol{K}_{u \boldsymbol{u}}^{-1}\left(\boldsymbol{s}\left[\boldsymbol{u}_{i}+\Delta \boldsymbol{u},\left(\lambda_{i}+\Delta \lambda\right) \overline{\boldsymbol{u}}\right]+\boldsymbol{K}_{\boldsymbol{u} \bar{u}} \dot{\lambda} \overline{\boldsymbol{u}}\right),
$$

${ }^{3}$ This orthogonality condition will be defined precisely below. 
once $\dot{\lambda}$ is known. Clearly, a further equation is needed in order to compensate for the new unknown $\dot{\lambda}$. We use to this aim the condition

$$
\left[\begin{array}{c}
\Delta \boldsymbol{u} \\
\Delta \lambda \overline{\boldsymbol{u}}
\end{array}\right] \cdot \boldsymbol{K}\left[\begin{array}{c}
\dot{\boldsymbol{u}} \\
\dot{\lambda} \overline{\boldsymbol{u}}
\end{array}\right]=0
$$

which expresses the orthogonality between $(\Delta \boldsymbol{u}, \Delta \lambda \overline{\boldsymbol{u}})$ and $(\dot{\boldsymbol{u}}, \dot{\lambda} \overline{\boldsymbol{u}})$ in the scalar product defined by the stiffness matrix $\boldsymbol{K}$ evaluated in $\left(\boldsymbol{u}_{i}+\Delta \boldsymbol{u}, \lambda_{i}+\Delta \lambda\right)$. Indeed, a simple computation gives

$$
\dot{\lambda}=-\frac{\Delta \boldsymbol{u} \cdot \boldsymbol{K}_{u u} \dot{\boldsymbol{u}}+\Delta \lambda \overline{\boldsymbol{u}} \cdot \boldsymbol{K}_{\bar{u} \boldsymbol{u}} \dot{\boldsymbol{u}}}{\Delta \boldsymbol{u} \cdot \boldsymbol{K}_{u \bar{u}} \overline{\boldsymbol{u}}+\Delta \lambda \overline{\boldsymbol{u}} \cdot \boldsymbol{K}_{\bar{u} \bar{u}} \overline{\boldsymbol{u}}}
$$

and by using (7), (9), and the definitions of $\hat{\boldsymbol{u}}$ and $\Delta \boldsymbol{u}$ in (5), we obtain

$$
\dot{\lambda}=\frac{\hat{\boldsymbol{u}} \cdot s+\bar{u} \cdot K_{\bar{u} u} K_{u u}^{-1} s}{\bar{u} \cdot\left(K_{\bar{u} \bar{u}}-K_{\bar{u} u} K_{u u}^{-1} K_{u \bar{u}}\right) \bar{u}},
$$

which is the correction for the extrapolation $\Delta \lambda$. Successively, by using (7), we can compute the correction $\dot{\boldsymbol{u}}$ for the Newton extrapolation $\Delta \boldsymbol{u}$. We remark that three terms can be distinguished in (10):

(1) The first addend of the numerator is the work done by the structural reaction $s\left[\boldsymbol{u}_{i}+\Delta \boldsymbol{u},\left(\lambda_{i}+\Delta \lambda\right) \overline{\boldsymbol{u}}\right]$ on displacements $\hat{\boldsymbol{u}}$ of the free nodes.

(2) The second addend of the numerator complements the above-mentioned work done by the structural reaction on free nodes with the work done by

$$
\boldsymbol{K}_{\bar{u} u} K_{u u}^{-1} s\left[u_{i}+\Delta u,\left(\lambda_{i}+\Delta \lambda\right) \bar{u}\right]
$$

on the constrained displacements $\overline{\boldsymbol{u}}$. In formulae we have that

$$
\boldsymbol{K}_{\bar{u} u} \boldsymbol{K}_{u u}^{-1} s=\bar{s}\left[u_{i}+\Delta u+K_{u u}^{-1} s,\left(\lambda_{i}+\Delta \lambda\right) \bar{u}\right]-\bar{s}\left[u_{i}+\Delta u,\left(\lambda_{i}+\Delta \lambda\right) \bar{u}\right],
$$

where $\bar{s}[\cdot]$ collects entries of the structural reaction corresponding to constrained displacements.

(3) The denominator can be interpreted as the work done by the structural reaction on the constrained displacements. In formulae we have that

$$
\begin{aligned}
& \left(\boldsymbol{K}_{\bar{u} \bar{u}}-\boldsymbol{K}_{\bar{u} u} \boldsymbol{K}_{u u}^{-1} \boldsymbol{K}_{u \bar{u}}\right) \overline{\boldsymbol{u}} \\
& \quad \approx \frac{\overline{\boldsymbol{s}}\left[\boldsymbol{u}_{i}+\Delta \boldsymbol{u},\left(\lambda_{i}+\Delta \lambda+\delta \lambda\right) \overline{\boldsymbol{u}}\right]-\overline{\boldsymbol{s}}\left[\boldsymbol{u}_{i}+\Delta \boldsymbol{u}+\delta \boldsymbol{u},\left(\lambda_{i}+\Delta \lambda\right) \overline{\boldsymbol{u}}\right]}{\delta \lambda},
\end{aligned}
$$

where $\delta \lambda=\alpha \Delta \lambda$, with $\alpha>0$ (good results are usually obtained using $\alpha=1)$, and

$$
\delta \boldsymbol{u}=\frac{1}{\delta \lambda} \boldsymbol{K}_{u \boldsymbol{u}}^{-1}\left(\boldsymbol{s}\left[\boldsymbol{u}_{i}+\Delta \boldsymbol{u},\left(\lambda_{i}+\Delta \lambda+\delta \lambda\right) \overline{\boldsymbol{u}}\right]-\boldsymbol{s}\left[\boldsymbol{u}_{i}+\Delta \boldsymbol{u},\left(\lambda_{i}+\Delta \lambda\right) \overline{\boldsymbol{u}}\right]\right) .
$$




\section{Numerical simulations}

Solving the Hencky model sketched in Section 2 by exploiting the algorithm briefly depicted in Section 3, we can compute equilibrium paths for pantographic beams. Numerical values employed for simulations are reported in Table 1. For all cases which are here studied, the length of the pantographic beam $L$ is the same. We analyzed pantographic beams with 21 (cases 1 and 3) and 41 (cases 2 and 4) unit cells. Cases 1 and 2 differ from cases 3 and 4 by the presence of additional rotational springs with stiffness $d$ (see Figure 1) on the left and right sides of the pantographic beam. In passing from case 1 to case 2 and from case 3 to case 4 , the number of unit cells $n_{\mathrm{uc}}$ is almost (due to symmetry reasons) doubled. In addition, in passing from case 1 to case 2 and from case 3 to case 4, we rescaled stiffnesses according to the following law used in [Barchiesi et al. 2018; 2019] for getting the homogenized continuum model as $n_{\mathrm{uc}} \rightarrow \infty$ for fixed total length $L$ :

$$
a \sim n_{\mathrm{uc}}^{3}, \quad b \sim n_{\mathrm{uc}}, \quad c \sim n_{\mathrm{uc}}^{-1}, \quad d \sim n_{\mathrm{uc}} .
$$

We notice that $\ell_{0}=\left\|P_{j}-P_{i}\right\|$ in (2) is equal to $\sqrt{2} /\left(2 n_{\mathrm{uc}}\right)$ (here, $P_{i}$ and $P_{j}$ are two pivots which share the same extensional spring). Therefore, the product $a \ell_{0}$, which will be used in the sequel to scale some quantities, depends upon $n_{\mathrm{uc}}$. We also remark that the scaling law (14) entails that $a / b \sim n_{\mathrm{uc}}^{2}$ and $b / c \sim n_{\mathrm{uc}}^{2}$. This means that for large values of $n_{\mathrm{uc}}$, complete cell closure in straight (i.e., pantographic mechanism) or acuminate geometry - depending upon cell position and boundary conditions - becomes especially favorable energetically for sufficiently large prescribed displacements

Case study 1. By using the number of unit cells and the values for the stiffnesses reported in the first line of Table 1, we computed the equilibrium path shown in Figure 2 (continuous black line), which is the locus of pairs made up of the value of the constrained displacement at midpoint, represented by the parameter $\lambda$, and the corresponding (global) reaction force $R$ at equilibrium.

\begin{tabular}{|cccccc|}
\hline case & $n_{\mathrm{uc}}$ & $a$ & $b$ & $c$ & $d$ \\
\hline 1 & 21 & 10000 & 10.000 & 0.025000 & 0 \\
2 & 41 & 74421 & 19.524 & 0.012805 & 0 \\
\hline 3 & 21 & 10000 & 10.000 & 0.025000 & 10.000 \\
4 & 41 & 74421 & 19.524 & 0.012805 & 19.524 \\
\hline
\end{tabular}

Table 1. Data, in SI units, used for simulations of a pantographic beam $L=1.05$ long. 


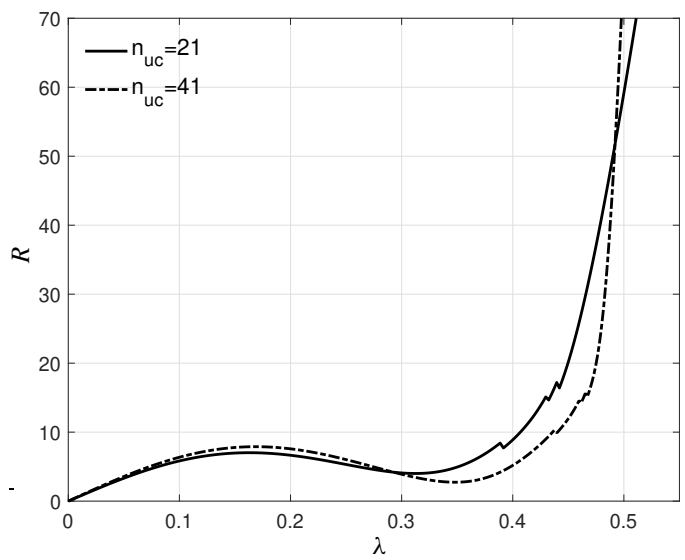

Figure 2. Equilibrium path, i.e., locus of pairs made up of the value of the constrained displacement, $\lambda$, at midpoint and the corresponding reaction force $R$ at equilibrium, of the pantographic beam for case 1 (continuous black line) and case 2 (dot-dashed black line).

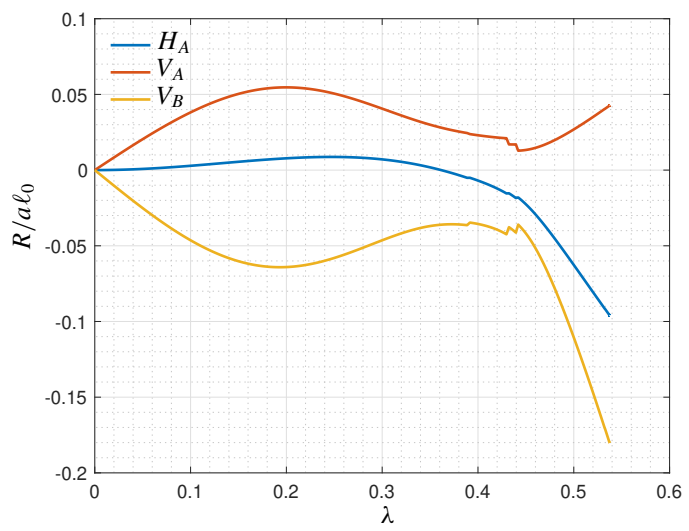

Figure 3. Scaled reactions corresponding to constraints enforced at nodes $A$ and $B$ ( $H$ and $V$ are the horizontal and vertical components, respectively) versus $\lambda$ (case 1 ).

In addition, we also plot in Figure 3 the reactions - scaled by the product $a \ell_{0}-$ corresponding to the constraints enforced at nodes $A$ and $B$ - placed on the left of the pantographic beam - versus the constrained vertical displacement $\lambda$ of the midnode of the structure.

We further plot in Figure 4 the total strain energy - scaled by the product $a \ell_{0}^{2}-$ versus the constrained vertical displacement $\lambda$ at the midnode of the structure. In the same figure we also plot the contributions to the total strain energy - scaled 


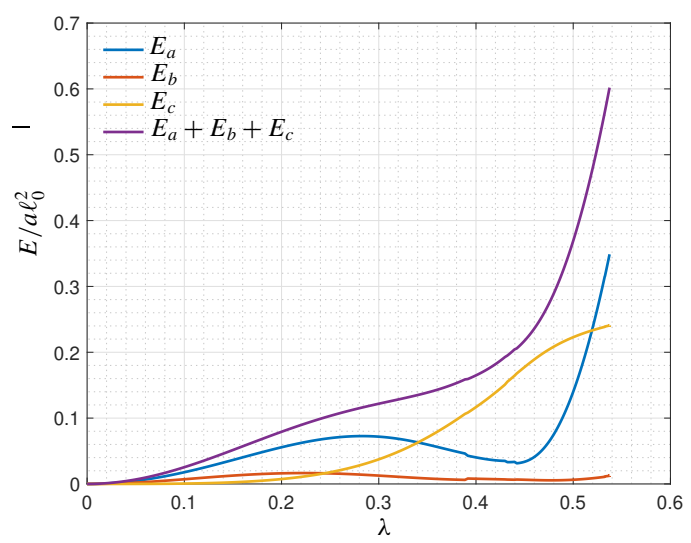

Figure 4. Scaled strain energies versus $\lambda$. Stretching $E_{a}$, bending $E_{b}$, and shearing $E_{c}$ contribute to total strain energy $E_{a}+E_{b}+E_{c}$ (case 1).

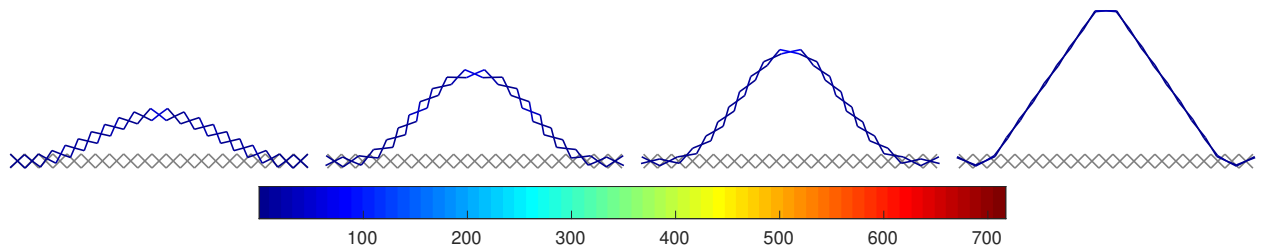

Figure 5. From left to right: deformation corresponding to the local maximum, the local minimum, the stage immediately after the first snap-through, and the last computed point of the equilibrium path reported in Figure 2 with a continuous black line (case 1). Colors indicate the strain energy.

again by the product $a \ell_{0}^{2}$ — as distinguished in stretching, bending, and shearing contributions.

Finally, we plot in Figure 5 the current configuration of the system for four values of the parameter $\lambda$ corresponding to (see Figure 2 (continuous black line)), the local maximum, i.e., limit point, the local minimum, the stage immediately after the first reaction force jump, and the last computed equilibrium point belonging to the increasing branch following the last reaction force jump.

Case study 2. Results for the case study 2 take into account a pantographic beam with 41 unit cells (previously, in case study 1, we used 21 unit cells). The parameters which were used are reported in the second line of Table 1 (stiffnesses in case 1 are rescaled according to (14)). The equilibrium path is plotted in Figure 2 (dot-dashed black line). The two curves in Figure 2 are very close and suggest that independence of the unit cell size $1 / n_{\mathrm{uc}}$ holds, thus indicating that the scaling 


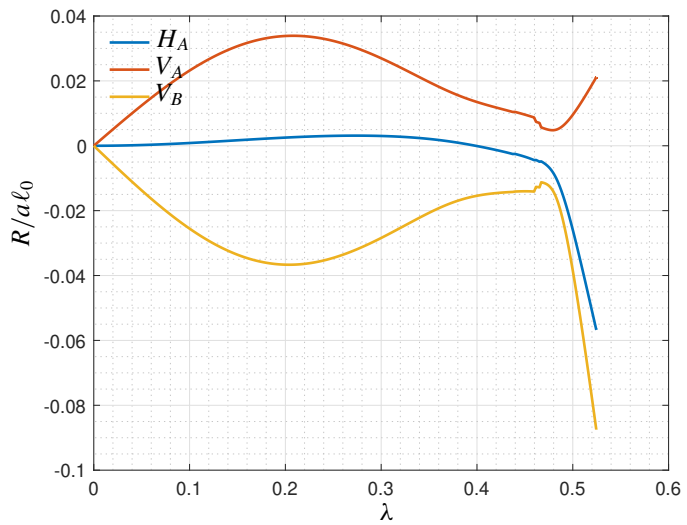

Figure 6. Scaled reactions corresponding to constraints enforced at nodes $A$ and $B$ ( $H$ and $V$ are the horizontal and vertical components, respectively), versus $\lambda$ (case 2 ).

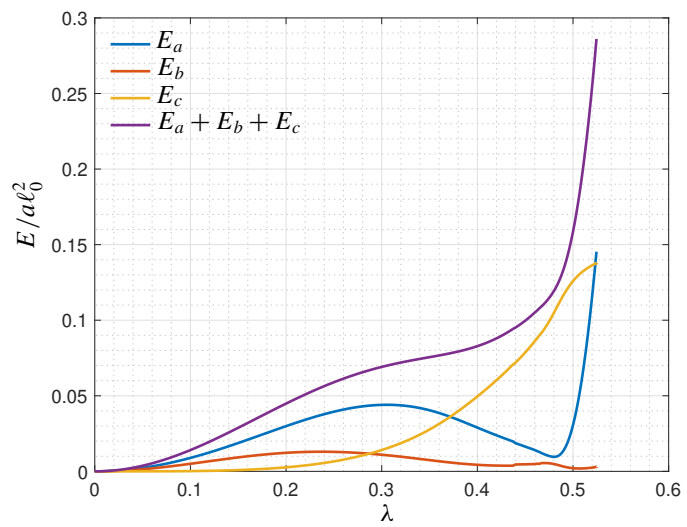

Figure 7. Scaled strain energies versus $\lambda$. Stretching $E_{a}$, bending $E_{b}$, and shearing $E_{c}$ contribute to total strain energy $E_{a}+E_{b}+E_{c}$ (case 2).

law employed for deriving the homogenized model implies the existence of a limit behavior. The reactions - scaled by the product $a \ell_{0}$ - corresponding to the constraints enforced at nodes $A$ and $B$ versus the constrained vertical displacement $\lambda$ of the midnode of the structure are plotted in Figure 6.

The total strain energy - scaled by the product $a \ell_{0}^{2}$ - versus the constrained vertical displacement $\lambda$ at the midnode of the structure is plotted in Figure 7. Contributions to the total strain energy - scaled again by the product $a \ell_{0}^{2}$ - as distinguished in stretching, bending, and shearing contributions are also plotted in the same figure. 


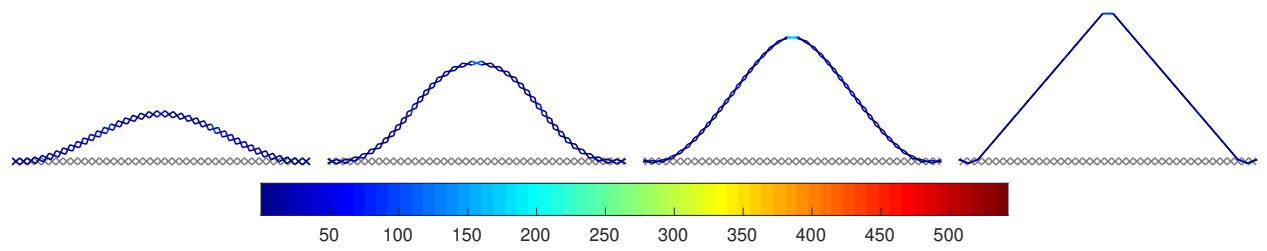

Figure 8. From left to right: deformation corresponding to the local maximum, the local minimum, the stage immediately after the first snap-through, and the last computed point of the equilibrium path reported in Figure 2 with a dot-dashed black line (case 2). Colors indicate the strain energy.

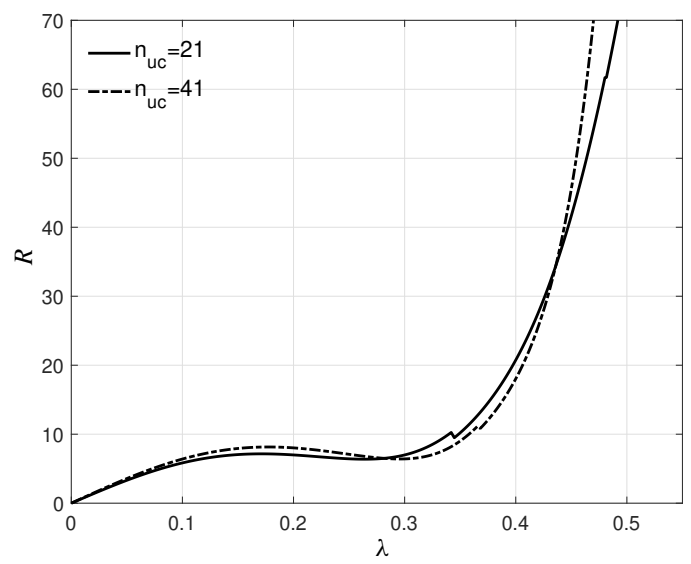

Figure 9. Equilibrium path, i.e., locus of pairs made up of the value of the constrained displacement at midpoint, $\lambda$, and the corresponding reaction force $R$ at equilibrium, of the pantographic beam for case 3 (continuous black line) and case 4 (dot-dashed black line).

Figure 8 shows the current configuration of the system for four values of the parameter $\lambda$ corresponding to (see Figure 2 (dot-dashed black line)) the local maximum, i.e., limit point, the local minimum, the stage immediately after the first reaction force jump, and the last computed equilibrium point belonging to the increasing branch following the last reaction force jump.

Case study 3. Results for the case study 3 take into account a pantographic beam with 21 unit cells. The parameters which were used are reported in the third line of Table 1. The equilibrium path is plotted in Figure 9 (continuous black line). 


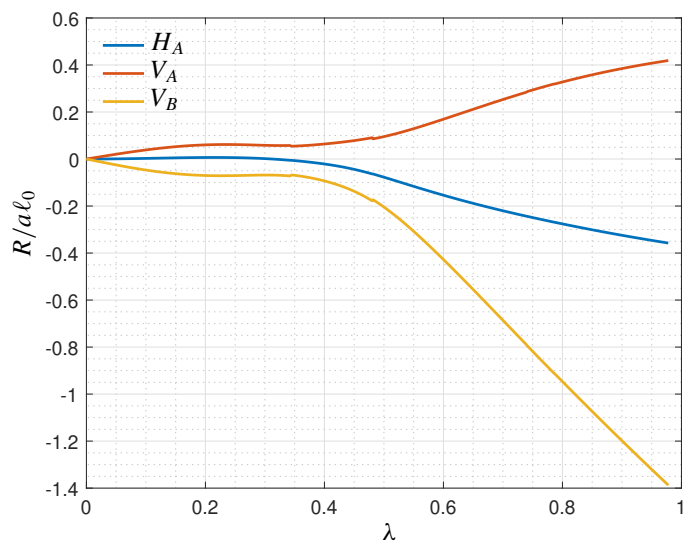

Figure 10. Scaled reactions corresponding to constraints enforced at nodes $A$ and $B$ ( $H$ and $V$ are the horizontal and vertical components, respectively) versus $\lambda$ (case 3 ).

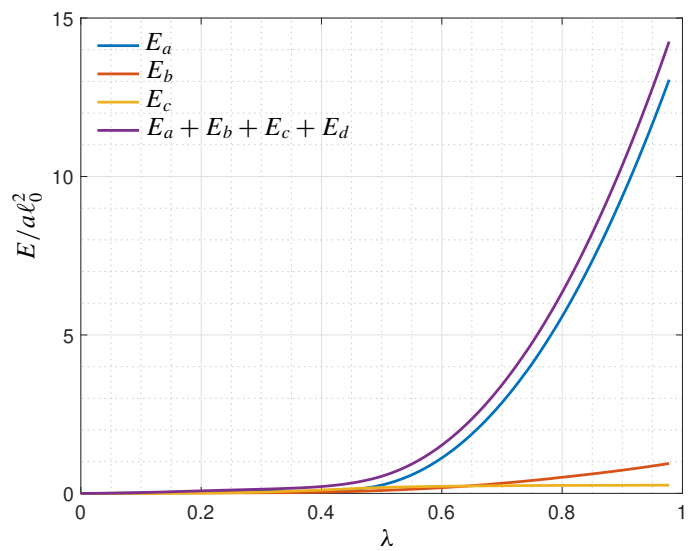

Figure 11. Scaled strain energies versus $\lambda$. Stretching $E_{a}$, bending $E_{b}$, and shearing $E_{c}$ contribute to total strain energy $E_{a}+E_{b}+$ $E_{c}+E_{d}$ for stiffness parameters (case 3).

The reactions - scaled by the product $a \ell_{0}$ - corresponding to the constraints enforced at nodes $A$ and $B$ versus the constrained vertical displacement $\lambda$ of the midnode of the structure are plotted in Figure 10.

The total strain energy - scaled by the product $a \ell_{0}^{2}$ - versus the constrained vertical displacement $\lambda$ at the midnode of the structure is plotted in Figure 11. Contributions to the total strain energy - scaled again by the product $a \ell_{0}^{2}$ - as distinguished in stretching, bending, and shearing contributions are also plotted in the same figure. 


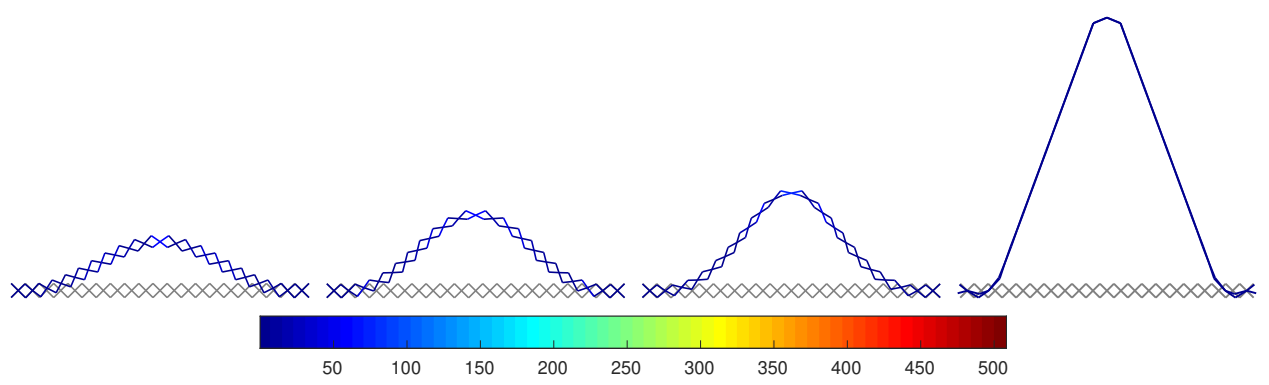

Figure 12. From left to right: deformation corresponding to the local maximum, the local minimum, the stage immediately after the first snap-through, and the last computed point of the equilibrium path reported in Figure 9 with a continuous black line (case 3).

Colors indicate the strain energy.

Figure 12 shows the current configuration of the system for four values of the parameter $\lambda$ corresponding to (see Figure 9 (continuous black line)) the local maximum, i.e., limit point, the local minimum, the stage immediately after the first reaction force jump, and the last computed equilibrium point belonging to the increasing branch following the last reaction force jump.

Case study 4. Results for the case study 4 take into account a pantographic beam with 41 unit cells (previously, in case study 3, we used 21 unit cells). The parameters which were used are reported in the fourth line of Table 1 (stiffnesses in case 3 are rescaled according to (14)). The equilibrium path is plotted in Figure 9 (dashed black line). Again, the curves in Figure 9 are very close and suggest that independence of the unit cell size $1 / n_{\mathrm{uc}}$ holds, thus indicating that the scaling law employed for deriving the homogenized model implies the existence of a limit behavior. The reactions - scaled by the product $a \ell_{0}$ - corresponding to the constraints enforced at nodes $A$ and $B$ versus the constrained vertical displacement $\lambda$ of the midnode of the structure are plotted in Figure 13.

The total strain energy - scaled by the product $a \ell_{0}^{2}$ - versus the constrained vertical displacement $\lambda$ at the midnode of the structure is plotted in Figure 14. Contributions to the total strain energy — scaled again by the product $a \ell_{0}^{2}$ - as distinguished in stretching, bending, and shearing contributions are also plotted in the same figure.

Figure 15 shows the current configuration of the system for four values of the parameter $\lambda$ corresponding to (see Figure 9 (dashed black line)) the local maximum, i.e., limit point, the local minimum, the stage immediately after the first reaction force jump, and the last computed equilibrium point belonging to the increasing branch following the last reaction force jump. 


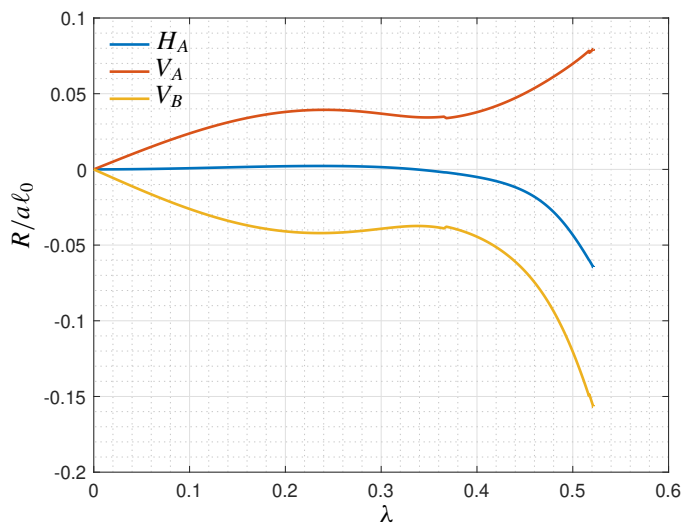

Figure 13. Scaled reactions corresponding to constraints enforced at nodes $A$ and $B$ ( $H$ and $V$ are the horizontal and vertical components, respectively) versus $\lambda$ (case 4 ).

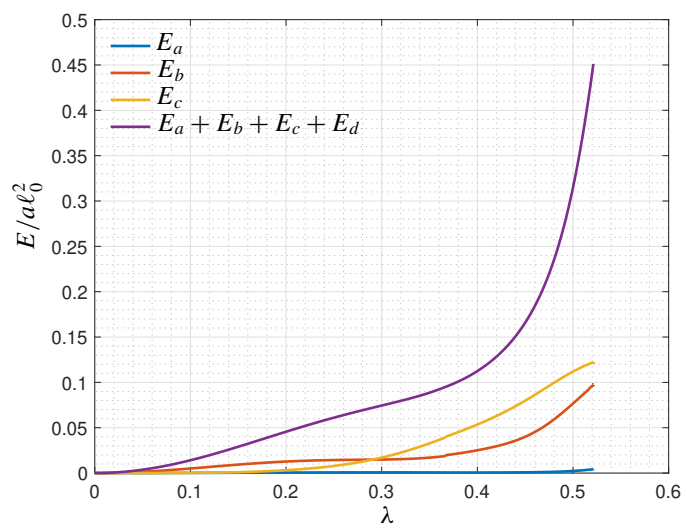

Figure 14. Scaled strain energies versus $\lambda$. Stretching $E_{a}$, bending $E_{b}$, and shearing $E_{c}$ contribute to total strain energy $E_{a}+E_{b}+$ $E_{c}+E_{d}$ (case 4$)$.

Sensitivity analysis respect to the stiffness parameters. In addition, we perform an analysis of the equilibrium path sensitivity with respect to changes in stiffness parameters of the pantographic beam subjected to the three-point bending test, which is reported in Figure 16. We observe that, basically, each of the found behaviors can be regarded as a degeneracy of the equilibrium path in Figure 2. The variety of obtained behaviors suggests that tuning of the pantographic beam mechanical response deserves future investigation.

Remarks on numerical simulations. We notice that: 




Figure 15. From left to right: deformation corresponding to the local maximum, the local minimum, the stage immediately after the first snap-through, and the last computed point of the equilibrium path reported in Figure 9 with a dot-dashed black line (case 4). Colors indicate the strain energy.


Figure 16. Sensitivity analysis of the equilibrium path respect to the stiffness parameters $a, b$, and $c$ for the case study 1 .

(1) In all cases, the force-displacement equilibrium paths, in continuous and dashed black line in Figures 2 and 9, show the existence of two limit points, i.e., the tangent to the equilibrium path is horizontal/vertical (horizontal in this case). When increasing the constrained displacement at midpoint, the presence of an increasing reaction branch in the equilibrium path followed by a 


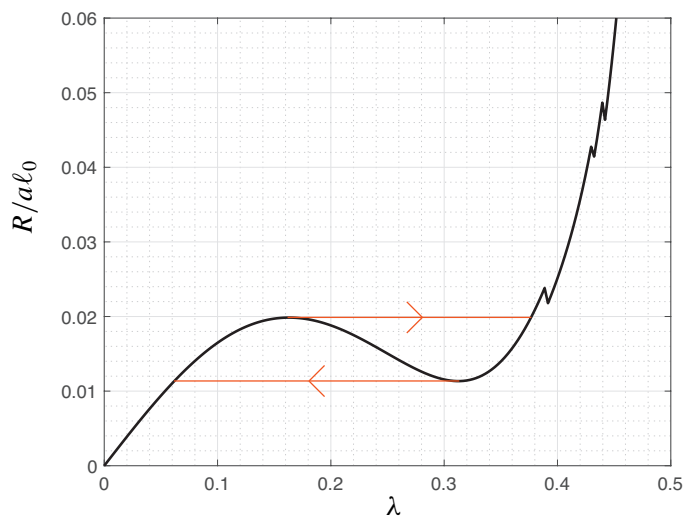

Figure 17. Equilibrium path for case study 1 with indication, in red, of the force-controlled loading-unloading pseudoelastic cycle.

decreasing one, passing through a limit point, gives a well known response (an analogous discussion can be given for the presence of a decreasing reaction branch followed by an increasing one, passing through a limit point). Indeed, it implies that, when considering a monotonically increasing prescribed force acting on the midpoint (therefore, we do not apply any more displacement but force), when the limit point is reached, then the equilibrium displacement experiences a sudden increase (see the red lines in Figure 17 indicating a forcecontrolled loading-unloading pseudoelastic cycle) [Eremeyev and Lebedev 2006]. We remark that such a phenomenon, which is observed in structures like the von Mises arch, contrarily to said structures, does not involve any local nor global snap-through instability, but it is a geometrical effect. Such an effect can be exploited in multiple applications requiring so-called mechanical switches.

(2) In all cases three local snap-through instabilities are occurring in the threepoint bending problem when varying the constrained vertical displacement, which are signaled in all plots (except for the deformed equilibrium configurations where they can be seen visually) by small bumps. The first snap-through involves the two cells adjacent to the midpoint, while the following two snapthrough instabilities occur for two different values of $\lambda$ at the boundary cells. These two last snap-through instabilities occur for higher values of $\lambda$ if $d$ increases, as $d$ is in that respect a penalization term.

(3) The horizontal reaction force $H$ in Figure 3 changes its sign with $\lambda$ in all cases.

(4) Looking at Figures 5, 8, 12, and 15, and particularly at points on the centerline of the structure (macroscopic deformed line), with increasing midnode 
constrained vertical displacement, for all cases, the structure switches progressively from a macroscopic flexural behavior to an extensional one. This is due to cells exploiting the pantographic mode to minimize the deformation energy of the structure. Such a behavior is further triggered at microscale by the occurrence of local snap-through instabilities. Indeed, cell closure in acuminate shape leads to progressively more and more localized macroscopic flexural deformations (which are unavoidable to fulfill kinematic constraints; in addition, as already mentioned, macroscopic flexure is not energetically penalized in such situation) in the vicinity of constrained nodes where snapthrough instabilities are occurring. In all cases, for the highest $\lambda$ of the computation, macroscopic flexural deformation is completely concentrated in cells adjacent to constrained nodes (see again Figures 5, 8, 12, and 15). Other regions of the beam are macroscopically straight and therefore experience only macroscopic extension. Straight closed cells in the current configuration store energy only in extensional springs. Clearly, the size of this localization depends upon the unit cell size and will degenerate at a point when $n_{\mathrm{uc}} \rightarrow \infty$, i.e., at macroscale in the homogenized continuum. It seems then reasonable to conjecture that, at macroscale, the beam will undergo at boundaries and at midpoint a beam-to-cable phase transition and assume for sufficiently large values of $\lambda$ a triangle shape. All these considerations are reflected in the equilibrium path, which shows with increasing midnode constrained vertical displacement - following the limit point — a decreasing reaction branch (due to not-yet-complete cell closure by pantographic mode) and (following complete cell closure) a successive stiffening controlled by extensional springs (which are according to rescaling law (14) the stiffest ones indeed). The scaled strain energies in Figures 4, 7, 11, and 14 clearly show that deformation energy stored within extensional springs increases superlinearly, reaching values comparable to those of energy stored within flexural-rotational springs at the end of the computation.

\section{Concluding remarks and future challenges}

It should at this point be clear why the results here reported are to be intended as an aid toward the formulation of conjectures attaining the continuum model and its related degeneracies. Especially, we were motivated in this investigation by understanding which features of the nonlinear behavior of slender pantographic discrete structure are - up to homogenization error (which clearly decreases with $n_{\mathrm{uc}}$ ) independent of the unit cell size $1 / n_{\mathrm{uc}}$ and should be observed in the homogenized continuum model. In this regard, it is worth noting that, in Figures 2 and 9, low sensitivity of the equilibrium path has been observed by increasing the cell number. 
Looking at the same figures (and at the other ones) one would conclude that bumps, which correspond to snap-throughs, disappear in the limit of the cell number going to infinity. Nevertheless, it remains an open question whether the observed bumps - i.e., reaction force/energy drop with respect to displacement increase will tend to have a (smaller) finite amplitude or not. More investigations in this regard are therefore required.

As we mentioned in the introduction, homogenized pantographic beams are complete second-gradient 1D-continua. Such continua have been previously validated by numerical discrete simulations based on Newton's method [Barchiesi et al. 2019]. Clearly, results reported herein should be regarded also as an extension of such previous work. Indeed, while solving the macromodel for prescribed (large) displacements up to an apparent negative stiffness regime - passing through the occurrence of limit points - was possible, that was up to now not possible for the micromodel.

In this respect, owing to the results obtained herein, we are able to state that simulations at microlevel confirm the predictions of the homogenized continuum. It is therefore possible to conclude that such negative stiffness effects and the occurrence of limit points in the equilibrium path, which are due to collective concurrence of geometric and mechanical properties of single elements (and not due to any local or global snap-through) arranged in pantographic topology, is mainly controlled at macroscale by second-gradient effects, i.e., at microscale by pantographic mechanism, as opposed to bending effects.

Finally, we list some challenges that surely will be tackled in the near future:

(1) The extension to 3D cases in order to take into account out-of-plane displacements; see [Giorgio et al. 2017b] for a continuum model able to predict outof-plane bifurcations and [Turco 2018a; Eugster et al. 2014] for a first attempt to model 3D inextensible beams using the Hencky guidelines.

(2) Some technical applications require removing the hypothesis of quasistatic application of external loads or given displacements; in these cases we have to consider inertia forces following the suggestions reported in [Giorgio et al. 2017a; Engelbrecht and Berezovski 2015; Tahaei Yaghoubi et al. 2018].

(3) Stiffness parameters of the pantographic beam have been chosen starting from estimates derived from global parameters of the continuum model; however, any methods capable of correlating experimental results with the stiffness parameters of the proposed model are surely interesting; see, e.g., [Placidi et al. 2015].

(4) Here we used simple laws to describe extensional, flexural, and shearing interaction; however, other interaction laws might be considered, e.g., those 
reported in [Braides and Solci 2016; Atai and Steigmann 1997; Challamel et al. 2014].

(5) Since the pantographic beam presented in the foregoing could be regarded as the representative volume element for continuum models and since, to obtain quantitative results in general problems, continuum models have to be discretized, it appears interesting to consider modern interpolations laws based on B-splines and NURBS; see, for an insight, [Greco et al. 2017; Greco and Cuomo 2013; Cuomo et al. 2014; Balobanov and Niiranen 2018].

(6) Hencky models are the fundamental building block for continuum models such as, e.g., those presented in [Khakalo et al. 2018; Seppecher 1996; Pideri and Seppecher 1997a; 1997b; Scala and Van Goethem 2016; Lurie and Kalamkarov 2007; Gusev and Lurie 2017; Giorgio 2016; Abali et al. 2016; Misra and Poorsolhjouy 2015; 2017; Shirani et al. 2019; De Masi et al. 2008; 2009; Carlen et al. 2009; Braides et al. 2009]; therefore, the developments of new and efficient Hencky models, besides to be useful to build reference results as shown in this paper, are surely a valuable contribution towards the construction of continuum models; see also [dell'Isola et al. 2017] for a historical framework and [Reiher et al. 2017] for some insight about the computational point of view.

(7) Even though the results presented here consider only elastic deformation patterns, it is possible to build Lagrangian models including damaging [Turco et al. 2016d]; furthermore, it is possible to include novel direct continuum modeling proposed in [Placidi and Barchiesi 2018; Giorgio and Scerrato 2017; Placidi et al. 2018; Shirani et al. 2017; Ganghoffer and Pastrone 2009].

\section{References}

[Abali et al. 2016] B. E. Abali, C.-C. Wu, and W. H. Müller, "An energy-based method to determine material constants in nonlinear rheology with applications", Continuum Mech. Therm. 28:5 (2016), 1221-1246.

[Alibert et al. 2003] J.-J. Alibert, P. Seppecher, and F. Dell'Isola, “Truss modular beams with deformation energy depending on higher displacement gradients", Math. Mech. Solids 8:1 (2003), $51-73$.

[Atai and Steigmann 1997] A. A. Atai and D. J. Steigmann, "On the nonlinear mechanics of discrete networks", Arch. Appl. Mech. 67:5 (1997), 303-319.

[Balobanov and Niiranen 2018] V. Balobanov and J. Niiranen, "Locking-free variational formulations and isogeometric analysis for the Timoshenko beam models of strain gradient and classical elasticity", Comput. Methods Appl. Mech. Eng. 339 (2018), 137-159.

[Barchiesi et al. 2018] E. Barchiesi, F. dell'Isola, M. Laudato, L. Placidi, and P. Seppecher, "A 1D continuum model for beams with pantographic microstructure: asymptotic micro-macro identification and numerical results", pp. 43-74 in Advances in mechanics of microstructured media and structures, edited by F. dell'Isola et al., Adv. Struct. Mater. 87, Springer, 2018. 
[Barchiesi et al. 2019] E. Barchiesi, S. R. Eugster, L. Placidi, and F. dell'Isola, "Pantographic beam: a complete second gradient 1D-continuum in plane", Z. Angew. Math. Phys. 70:5 (2019), art. id. 135.

[Bertoldi et al. 2017] K. Bertoldi, V. Vitelli, J. Christensen, and M. van Hecke, "Flexible mechanical metamaterials", Nat. Rev. Mater. 2 (2017), art. id. 17066.

[Braides and Solci 2016] A. Braides and M. Solci, "Asymptotic analysis of Lennard-Jones systems beyond the nearest-neighbour setting: a one-dimensional prototypical case", Math. Mech. Solids 21:8 (2016), 915-930.

[Braides et al. 2009] A. Braides, G. Riey, and M. Solci, "Homogenization of Penrose tilings", C. R. Math. Acad. Sci. Paris 347:11-12 (2009), 697-700.

[Carlen et al. 2009] E. A. Carlen, M. C. Carvalho, R. Esposito, J. L. Lebowitz, and R. Marra, "Droplet minimizers for the Gates-Lebowitz-Penrose free energy functional", Nonlinearity 22:12 (2009), 2919-2952.

[Challamel et al. 2014] N. Challamel, J. Lerbet, and C. M. Wang, "On buckling of granular columns with shear interaction: discrete versus nonlocal approaches", J. Appl. Phys. 115:23 (2014), art. id. 234902.

[Cuomo et al. 2014] M. Cuomo, L. Contrafatto, and L. Greco, "A variational model based on isogeometric interpolation for the analysis of cracked bodies”, Int. J. Eng. Sci. 80 (2014), 173-188.

[De Masi et al. 2008] A. De Masi, I. Merola, E. Presutti, and Y. Vignaud, "Potts models in the continuum: uniqueness and exponential decay in the restricted ensembles", J. Stat. Phys. 133:2 (2008), 281-345.

[De Masi et al. 2009] A. De Masi, I. Merola, E. Presutti, and Y. Vignaud, "Coexistence of ordered and disordered phases in Potts models in the continuum", J. Stat. Phys. 134:2 (2009), 243-306.

[dell' Isola et al. 2016] F. dell'Isola, I. Giorgio, M. Pawlikowski, and N. L. Rizzi, "Large deformations of planar extensible beams and pantographic lattices: heuristic homogenization, experimental and numerical examples of equilibrium”, P. Roy. Soc. A Math. Phys. 472:2185 (2016), art. id. 20150790.

[dell'Isola et al. 2017] F. dell'Isola, A. Della Corte, and I. Giorgio, "Higher-gradient continua: the legacy of Piola, Mindlin, Sedov and Toupin and some future research perspectives", Math. Mech. Solids 22:4 (2017), 852-872.

[dell'Isola et al. 2019a] F. dell'Isola, P. Seppecher, J. J. Alibert, T. Lekszycki, R. Grygoruk, M. Pawlikowski, D. Steigmann, I. Giorgio, U. Andreaus, E. Turco, M. Gołaszewski, N. Rizzi, C. Boutin, V. A. Eremeyev, A. Misra, L. Placidi, E. Barchiesi, L. Greco, M. Cuomo, A. Cazzani, A. Della Corte, A. Battista, D. Scerrato, I. Zurba Eremeeva, Y. Rahali, J.-F. Ganghoffer, W. Müller, G. Ganzosch, M. Spagnuolo, A. Pfaff, K. Barcz, K. Hoschke, J. Neggers, and F. Hild, "Pantographic metamaterials: an example of mathematically driven design and of its technological challenges", Continuum Mech. Therm. 31:4 (2019), 851-884.

[dell'Isola et al. 2019b] F. dell'Isola, P. Seppecher, M. Spagnuolo, E. Barchiesi, F. Hild, T. Lekszycki, I. Giorgio, L. Placidi, U. Andreaus, M. Cuomo, S. R. Eugster, A. Pfaff, K. Hoschke, R. Langkemper, E. Turco, R. Sarikaya, A. Misra, M. De Angelo, F. D’Annibale, A. Bouterf, X. Pinelli, A. Misra, B. Desmorat, M. Pawlikowski, C. Dupuy, D. Scerrato, P. Peyre, M. Laudato, L. Manzari, P. Göransson, C. Hesch, S. Hesch, P. Franciosi, J. Dirrenberger, F. Maurin, Z. Vangelatos, C. Grigoropoulos, V. Melissinaki, M. Farsari, W. Müller, B. Emek Abali, C. Liebold, G. Ganzosch, P. Harrison, R. Drobnicki, L. Igumnov, F. Alzahrani, and T. Hayat, "Advances in pantographic structures: design, manufacturing, models, experiments and image analyses", Continuum Mech. Therm. 31:4 (2019), 1231-1282. 
[dell'Isola et al. 2019c] F. dell'Isola, E. Turco, A. Misra, Z. Vangelatos, C. Grigoropoulos, V. Melissinaki, and M. Farsari, "Force-displacement relationship in micro-metric pantographs: experiments and numerical simulations”, C. R. Mécanique 347:5 (2019), 397-405.

[Engelbrecht and Berezovski 2015] J. Engelbrecht and A. Berezovski, "Reflections on mathematical models of deformation waves in elastic microstructured solids", Math. Mech. Complex Syst. 3:1 (2015), 43-82.

[Eremeyev and Lebedev 2006] V. A. Eremeyev and L. P. Lebedev, "On the loss stability of von Mises truss with the effect of pseudo-elasticity", Mat. Enseñ. Univ. (N. S.) 14:2 (2006), 111-118.

[Eugster et al. 2014] S. R. Eugster, C. Hesch, P. Betsch, and C. Glocker, "Director-based beam finite elements relying on the geometrically exact beam theory formulated in skew coordinates", Int. J. Numer. Methods Eng. 97:2 (2014), 111-129.

[Filipov et al. 2015] E. T. Filipov, T. Tachi, and G. H. Paulino, "Origami tubes assembled into stiff, yet reconfigurable structures and metamaterials", P. Natl. Acad. Sci. USA 112:40 (2015), 1232112326.

[Ganghoffer and Pastrone 2009] J.-F. Ganghoffer and F. Pastrone (editors), Mechanics of microstructured solids: cellular materials, fibre reinforced solids and soft tissues, Lecture Notes in Applied and Computational Mechanics 46, Springer, 2009.

[Giorgio 2016] I. Giorgio, "Numerical identification procedure between a micro-Cauchy model and a macro-second gradient model for planar pantographic structures", Z. Angew. Math. Phys. 67:4 (2016), art. id. 95.

[Giorgio and Scerrato 2017] I. Giorgio and D. Scerrato, "Multi-scale concrete model with ratedependent internal friction”, Eur. J. Environ. Civ. Eng. 21:7-8 (2017), 821-839.

[Giorgio et al. 2017a] I. Giorgio, A. Della Corte, and F. dell'Isola, "Dynamics of 1D nonlinear pantographic continua", Nonlinear Dynam. 88:1 (2017), 21-31.

[Giorgio et al. 2017b] I. Giorgio, N. L. Rizzi, and E. Turco, "Continuum modelling of pantographic sheets for out-of-plane bifurcation and vibrational analysis", P. Roy. Soc. A Math. Phys. 473:2207 (2017), art. id. 20170636.

[Greco and Cuomo 2013] L. Greco and M. Cuomo, "B-spline interpolation of Kirchhoff-Love space rods”, Comput. Methods Appl. Mech. Eng. 256 (2013), 251-269.

[Greco et al. 2017] L. Greco, M. Cuomo, L. Contrafatto, and S. Gazzo, "An efficient blended mixed B-spline formulation for removing membrane locking in plane curved Kirchhoff rods", Comput. Methods Appl. Mech. Eng. 324 (2017), 476-511.

[Gusev and Lurie 2017] A. A. Gusev and S. A. Lurie, "Symmetry conditions in strain gradient elasticity", Math. Mech. Solids 22:4 (2017), 683-691.

[Khakalo et al. 2018] S. Khakalo, V. Balobanov, and J. Niiranen, "Modelling size-dependent bending, buckling and vibrations of 2D triangular lattices by strain gradient elasticity models: applications to sandwich beams and auxetics", Int. J. Eng. Sci. 127 (2018), 33-52.

[Lurie and Kalamkarov 2007] S. A. Lurie and A. L. Kalamkarov, "General theory of continuous media with conserved dislocations”, Int. J. Solids Struct. 43:22-23 (2007), 7468-7485.

[Misra and Poorsolhjouy 2015] A. Misra and P. Poorsolhjouy, "Identification of higher-order elastic constants for grain assemblies based upon granular micromechanics", Math. Mech. Complex Syst. 3:3 (2015), 285-308.

[Misra and Poorsolhjouy 2017] A. Misra and P. Poorsolhjouy, "Elastic behavior of 2D grain packing modeled as micromorphic media based on granular micromechanics", J. Eng. Mech. 143:1 (2017), art. id. C4016005. 
[Pideri and Seppecher 1997a] C. Pideri and P. Seppecher, "A second gradient material resulting from the homogenization of an heterogeneous linear elastic medium", Continuum Mech. Therm. 9:5 (1997), 241-257.

[Pideri and Seppecher 1997b] C. Pideri and P. Seppecher, "Un résultat d'homogénéisation pour un matériau élastique renforcé périodiquement par des fibres élastiques de très grande rigidité", $C$. $R$. Acad. Sci. IIB Mec. 324:8 (1997), 475-481.

[Placidi and Barchiesi 2018] L. Placidi and E. Barchiesi, "Energy approach to brittle fracture in strain-gradient modelling”, P. Roy. Soc. A Math. Phys. 474:2210 (2018), art. id. 20170878.

[Placidi et al. 2015] L. Placidi, U. Andreaus, A. D. Corte, and T. Lekszycki, "Gedanken experiments for the determination of two-dimensional linear second gradient elasticity coefficients", Z. Angew. Math. Phys. 66:6 (2015), 3699-3725.

[Placidi et al. 2018] L. Placidi, E. Barchiesi, and A. Misra, "A strain gradient variational approach to damage: a comparison with damage gradient models and numerical results", Math. Mech. Complex Syst. 6:2 (2018), 77-100.

[Reiher et al. 2017] J. C. Reiher, I. Giorgio, and A. Bertram, "Finite-element analysis of polyhedra under point and line forces in second-strain gradient elasticity", J. Eng. Mech. 143:2 (2017), art. id. 04016112.

[Riks 1972] E. Riks, “The application of Newton's method to the problem of elastic stability", $J$. Appl. Mech. T. ASME 39:4 (1972), 1060-1065.

[Scala and Van Goethem 2016] R. Scala and N. Van Goethem, "Constraint reaction and the PeachKoehler force for dislocation networks”, Math. Mech. Complex Syst. 4:2 (2016), 105-138.

[Seppecher 1996] P. Seppecher, "Moving contact lines in the Cahn-Hilliard theory", Int. J. Eng. Sci. 34:9 (1996), 977-992.

[Shirani et al. 2017] M. Shirani, M. T. Andani, M. Kadkhodaei, and M. Elahinia, "Effect of loading history on phase transition and martensitic detwinning in shape memory alloys: limitations of current approaches and development of a 1D constitutive model", J. Alloy. Comp. 729 (2017), 390-406.

[Shirani et al. 2019] M. Shirani, C. Luo, and D. J. Steigmann, "Cosserat elasticity of lattice shells with kinematically independent flexure and twist", Continuum Mech. Therm. 31:4 (2019), 10871097.

[Tahaei Yaghoubi et al. 2018] S. Tahaei Yaghoubi, V. Balobanov, S. M. Mousavi, and J. Niiranen, "Variational formulations and isogeometric analysis for the dynamics of anisotropic gradient-elastic Euler-Bernoulli and shear-deformable beams", Eur. J. Mech. A Solids 69 (2018), 113-123.

[Turco 2018a] E. Turco, "Discrete is it enough? The revival of Piola-Hencky keynotes to analyze three-dimensional elastica", Continuum Mech. Therm. 30:5 (2018), 1039-1057.

[Turco 2018b] E. Turco, "In-plane shear loading of granular membranes modeled as a Lagrangian assembly of rotating elastic particles", Mech. Res. Commun. 92 (2018), 61-66.

[Turco and Rizzi 2016] E. Turco and N. L. Rizzi, "Pantographic structures presenting statistically distributed defects: numerical investigations of the effects on deformation fields", Mech. Res. Commun. 77 (2016), 65-69.

[Turco et al. 2016a] E. Turco, K. Barcz, M. Pawlikowski, and N. L. Rizzi, "Non-standard coupled extensional and bending bias tests for planar pantographic lattices, I: numerical simulations", $Z$. Angew. Math. Phys. 67:5 (2016), art. id. 122.

[Turco et al. 2016b] E. Turco, K. Barcz, and N. L. Rizzi, "Non-standard coupled extensional and bending bias tests for planar pantographic lattices, II: comparison with experimental evidence", $Z$. Angew. Math. Phys. 67:5 (2016), art. id. 123. 
[Turco et al. 2016c] E. Turco, F. dell'Isola, A. Cazzani, and N. L. Rizzi, "Hencky-type discrete model for pantographic structures: numerical comparison with second gradient continuum models", Z. Angew. Math. Phys. 67:4 (2016), art. id. 85.

[Turco et al. 2016d] E. Turco, F. dell'Isola, N. L. Rizzi, R. Grygoruk, W. H. Müller, and C. Liebold, "Fiber rupture in sheared planar pantographic sheets: numerical and experimental evidence", Mech. Res. Commun. 76 (2016), 86-90.

[Turco et al. 2016e] E. Turco, M. Golaszewski, A. Cazzani, and N. L. Rizzi, "Large deformations induced in planar pantographic sheets by loads applied on fibers: experimental validation of a discrete Lagrangian model”, Mech. Res. Commun. 76 (2016), 51-56.

[Turco et al. 2017a] E. Turco, I. Giorgio, A. Misra, and F. dell'Isola, "King post truss as a motif for internal structure of (meta)material with controlled elastic properties", Roy. Soc. Open Sci. 4:10 (2017), art. id. 171153.

[Turco et al. 2017b] E. Turco, M. Golaszewski, I. Giorgio, and F. D'Annibale, "Pantographic lattices with non-orthogonal fibres: experiments and their numerical simulations", Compos. B Eng. 118 (2017), 1-14.

[Turco et al. 2018] E. Turco, A. Misra, M. Pawlikowski, F. dell'Isola, and F. Hilde, "Enhanced PiolaHencky discrete models for pantographic sheets with pivots without deformation energy: numerics and experiments", Int. J. Solids Struct. 147 (2018), 94-109.

[Turco et al. 2019a] E. Turco, F. dell'Isola, and A. Misra, "A nonlinear Lagrangian particle model for grains assemblies including grain relative rotations", Int. J. Numer. Anal. Met. 43:5 (2019), 1051-1079.

[Turco et al. 2019b] E. Turco, A. Misra, R. Sarikaya, and T. Lekszycki, "Quantitative analysis of deformation mechanisms in pantographic substructures: experiments and modeling”, Continuum Mech. Therm. 31:1 (2019), 209-223.

Received 3 Jul 2019. Accepted 1 Dec 2019.

EMILIO TURCO: emilio.turco@uniss.it

Dipartimento di Architettura, Design e Urbanistica, Università degli Studi di Sassari, Sassari, Italy

EMILIO BARCHIESI: barchiesiemilio@gmail.com

Dipartimento di Ingegneria Strutturale e Geotecnica, Università degli Studi di Roma "La Sapienza", Roma, Italy 


\title{
A TWO-DIMENSIONAL CONTINUUM MODEL OF PANTOGRAPHIC SHEETS MOVING IN A 3D SPACE AND ACCOUNTING FOR THE OFFSET AND RELATIVE ROTATIONS OF THE FIBERS
}

\author{
IVAN GIORgIO, NiCOLA L. RizZI, \\ Ugo Andreaus and David J. SteigmanN
}

\begin{abstract}
Recently growing attention has been paid to the particular class of metamaterials which has been called pantographic. Pantographic metamaterials have the following peculiar features: (i) their continuum model, at the macroscale, has to include a term of the deformation energy depending on the second gradient of placement, (ii) they can show an elastic behavior in large deformation regimes, and (iii) they are resilient and tough during rupture phenomena (dell' Isola et al. 2015). In order to predict pantographic metamaterials' mechanical behavior, it is possible to introduce a three-dimensional continuum micromodel, in which their internal geometrical microstructure is described in detail. However, the computational costs of this choice are presently prohibitive. In this paper, we introduce a reduced order model for pantographic sheets - which are an example of an elastic surface - whose kinematics include, for each of the two constituting families of fibers fully independent three-dimensional (i) placement and (ii) rotation fields. In this way it is possible to include, also in the reduced order model, (i) the initial and the actual offset between the fibers and (ii) the deformation energy of the interconnecting pivots. By postulating a simplified expression for the deformation energy we prove that also a reduced order model can describe some experimental observed buckling and postbuckling phenomena. The promising results which we present here motivate the quest of more general expressions for deformation energy capable of capturing the fully nonlinear behavior exhibited by pantographic sheets.
\end{abstract}

\section{Introduction}

Metamaterials are materials with microstructures that have been designed to achieve novel macroscopic behavior tailored to some specific uses or having some specific

\section{Communicated by Victor A. Eremeyev.}

Giorgio is the corresponding author.

MSC2010: 74KXX, 74QXX.

Keywords: elastic surface theory, second-gradient models, pantographic structures. 




Figure 1. A sample of pantographic material.

features [Barchiesi et al. 2019b; dell'Isola et al. 2019]. In particular, pantographic metamaterials [Alibert et al. 2003; Seppecher et al. 2011; Eugster et al. 2019] possess some very peculiar characteristics. At the macroscale they entail deformation energies that typically depend only on the second gradient of placement. Pantographic metamaterials having deformation energies depending "mainly" on the second gradient of placement [Alibert and Della Corte 2019] are capable of large deformations that remain in an elastic regime and exhibit great toughness in rupture phenomena [Placidi et al. 2016; Turco et al. 2016b; De Angelo et al. $2019 \mathrm{~b}$ ]. Of course, to predict the behavior of pantographic metamaterials, it is possible to introduce a micromodel, where their internal microstructured architecture is fully described, in all its geometrical and mechanical complexity, by a conventional three-dimensional continuum. It is clear, on the other hand, that the computational burden implied by this modeling choice can be prohibitive, the availability of modern computing tools notwithstanding. Indeed, despite having optimized codes of this kind [Giorgio 2016], it is not feasible to find the solutions of structural optimization problems by employing, as subroutines, the codes calculating the equilibrium shapes of aforementioned three-dimensional continua. Therefore, the question arises: can we find a reduced order model with sufficient resolution and which is computationally convenient?

A first simplification can be achieved by limiting our attention to two-dimensional pantographic metamaterials, called also pantographic sheets. This has been considered in a series of papers [dell'Isola et al. 2015; 2019; Eremeyev et al. 2018; Barchiesi and Placidi 2017; Steigmann and dell'Isola 2015; Andreaus et al. 2018] where a specific class of two-dimensional continua, generalizing standard KirchhoffLove plate theory, is considered. In these models, the deformation energy depends not only on the second gradient of the transverse component of displacement, as in standard plate theory, but also on the second gradient of in-plane displacement.

Nevertheless, despite these advances, such models do not take into account some important mechanical features of pantographic sheets.

In particular, as shown in [De Angelo et al. 2019a], the mechanical behavior of pantographic sheets is affected by the deformation of the interconnecting pivots (see Figure 1) and on the (deformation-dependent) offset between the constituent fibers [Barchiesi et al. 2019a]. This phenomenology has yet to be modeled explicitly, 



Figure 2. Schematic representation of a connection: "lefthanded" and "right-handed", respectively.

except in the case of purely planar deformation [Spagnuolo et al. 2017]. On the other hand, it has been estimated, by using complex numerical simulations based on three-dimensional continuum modeling, that there is a large amount of deformation energy which is stored in pivots and nearby material points [Giorgio 2016]. Moreover, in some deformation processes, the offset between the fibers, together with their relative rotations, play a significant role in buckling and postbuckling phenomena. This suggests a form of chirality of pantographic sheets, i.e., some sheets are "left-handed" while others are "right-handed", due to the offset of constituent fibers (see Figure 2) and to the relative orientation of upper and lower arrays of fibers.

The aim of this paper is to present a reduced order model for pantographic sheets that accounts for the interplay among chirality, offset between the constituent fibers, and the deformation of interconnecting pivots play.

The reduced order continuum model for pantographic sheets discussed in this work has enriched kinematics, including:

(1) two distinct placement fields, one for each fiber family (the upper and the lower) constituting the sheet,

(2) two distinct rotation fields, describing the rotations of the sections of each family of fibers.

The enriched kinematics allows us to include, in the proposed "richer" reduced order model:

(1) the initial and the actual offset between the upper and lower families of fibers,

(2) the deformation energy stored in the interconnecting pivots.

The importance of these two mechanical properties of pantographic sheets has been emphasized in [Giorgio 2016] where it is proved, using simulations based on a detailed 3D first-gradient microcontinuum model, that in many deformation processes the separation distance of the fibers varies significantly and that a large percentage of deformation energy is stored in the small volumes containing the interconnecting pivots.

The reduced order model is then completed by postulating an expression for the deformation energy in terms of those deformation measures which are naturally introduced in terms of the proposed kinematical descriptors. 
Further, we postulate a simple expression for the deformation energy of the pivots which is physically well motivated when pivots undergo small deformations. By using this simplified deformation energy we show that the reduced order model can describe, at least in a qualitative way, the buckling and postbuckling phenomena that have been observed experimentally and predicted numerically.

To this end we compare the predictions of the present model with numerical simulations based on a refined three-dimensional continuum model, obtained with codes and numerical procedures which are further optimized relative to those used in [Giorgio 2016; De Angelo et al. 2019a; Cazzani et al. 2018a; 2018b].

The quantitative agreement is lost when interconnecting pivots undergo large deformations. This circumstance leads us to postulate more complex expressions for the deformation energy. Indeed our results suggest the structure and the properties of more general constitutive relations for the deformation energy aimed at describing the fully nonlinear behavior exhibited by pantographic sheets.

\section{Fiber kinematics}

Figure 3 depict the fiber directions in the pantographic sheet together with the local bases. In what follows all the components will be given in the global base $\boldsymbol{b}_{i}$. To describe fibers kinematics we introduce the fields

$$
\begin{array}{ll}
\text { fiber }_{1}: & { }^{1} \boldsymbol{u},{ }^{1} \boldsymbol{R}, \\
\text { fiber }_{2}: & { }^{2} \boldsymbol{u},{ }^{2} \boldsymbol{R},
\end{array}
$$

where the vector fields ${ }^{1} \boldsymbol{u}$ and ${ }^{2} \boldsymbol{u}$ are the displacements of the beam axes, and ${ }^{1} \boldsymbol{R}$ and ${ }^{2} \boldsymbol{R}$ are orthogonal tensor fields that represent the rotations of the beam crosssections (for a more comprehensive discussion, see [Eremeyev and Pietraszkiewicz 2016; 2006; Eremeyev 2018; 2019]). The components of these fields are

$$
\begin{array}{cc}
{ }^{1} \boldsymbol{u}={ }^{1} u_{i} \boldsymbol{b}_{i}, & { }^{2} \boldsymbol{u}={ }^{2} u_{i} \boldsymbol{b}_{i}, \\
{ }^{1} \boldsymbol{R}={ }^{1} R_{i j} \boldsymbol{b}_{i} \otimes \boldsymbol{b}_{j}, \quad{ }^{2} \boldsymbol{R}={ }^{2} \widetilde{R}_{i j} \boldsymbol{c}_{i} \otimes \boldsymbol{c}_{j}={ }^{2} R_{i j} \boldsymbol{b}_{i} \otimes \boldsymbol{b}_{j} .
\end{array}
$$

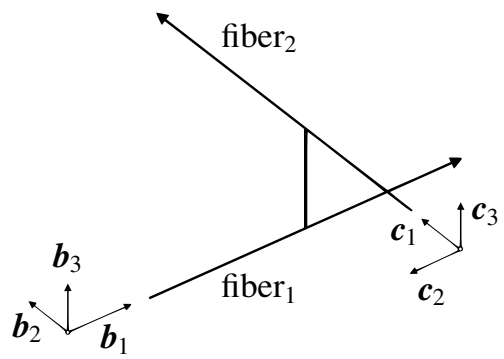

Figure 3. Reference frames. 
The components ${ }^{1} R_{i j}$ are given in terms of the Bryant angles ${ }^{1} \varphi_{1},{ }^{1} \varphi_{2}$, and ${ }^{1} \varphi_{3}$ and ${ }^{2} \widetilde{R}_{i j}$ are given in terms of ${ }^{2} \varphi_{1},{ }^{2} \varphi_{2}$, and ${ }^{2} \varphi_{3}$.

The deformation measures are

$$
\begin{aligned}
{ }^{\alpha} \boldsymbol{e} & ={ }^{\alpha} \boldsymbol{R}^{\top}\left({ }^{\alpha} \boldsymbol{X}^{\prime}+{ }^{\alpha} \boldsymbol{u}^{\prime}\right)-{ }^{\alpha} \boldsymbol{X}^{\prime}, \\
{ }^{\alpha} \boldsymbol{\kappa} & =\left({ }^{\alpha} \boldsymbol{R}^{\top \alpha} \boldsymbol{R}^{\prime}\right)_{\times},
\end{aligned}
$$

where $\alpha=\{1,2\},{ }^{\alpha} \boldsymbol{X}$ are the position of the beam axes in the reference configuration, the prime is used to denote differentiation with respect to the abscissa along the axes, and ${ }^{\alpha} \boldsymbol{\kappa}$ is the axial vector of the skew tensor ${ }^{\alpha} \boldsymbol{R}^{\top \alpha}{ }^{\prime} \boldsymbol{R}^{\prime}$.

The components of ${ }^{\alpha} \boldsymbol{e}$ and ${ }^{\alpha} \boldsymbol{\kappa}$ in the global basis are

$$
\begin{aligned}
& { }^{1} \varepsilon={ }^{1} e_{1}=-1+\left(1+{ }^{1} u_{1,1}\right) \cos { }^{1} \varphi_{2} \cos { }^{1} \varphi_{3} \\
& +{ }^{1} u_{2,1}\left(\cos { }^{1} \varphi_{3} \sin ^{1} \varphi_{1} \sin ^{1} \varphi_{2}+\cos { }^{1} \varphi_{1} \sin ^{1} \varphi_{3}\right) \\
& +{ }^{1} u_{3,1}\left(\sin ^{1} \varphi_{1} \sin ^{1} \varphi_{3}-\cos ^{1} \varphi_{1} \cos { }^{1} \varphi_{3} \sin ^{1} \varphi_{2}\right), \\
& { }^{1} \gamma_{p}={ }^{1} e_{2}=-\left(1+{ }^{1} u_{1,1}\right) \cos { }^{1} \varphi_{2} \sin ^{1} \varphi_{3} \\
& +{ }^{1} u_{2,1}\left(\cos { }^{1} \varphi_{1} \cos { }^{1} \varphi_{3}-\sin ^{1} \varphi_{1} \sin ^{1} \varphi_{2} \sin ^{1} \varphi_{3}\right) \\
& +{ }^{1} u_{3,1}\left(\cos { }^{1} \varphi_{3} \sin ^{1} \varphi_{1}+\cos ^{1} \varphi_{1} \sin ^{1} \varphi_{2} \sin ^{1} \varphi_{3}\right) \text {, } \\
& { }^{1} \gamma_{n}={ }^{1} e_{3}=\left(1+{ }^{1} u_{1,1}\right) \sin { }^{1} \varphi_{2}-{ }^{1} u_{2,1} \cos { }^{1} \varphi_{2} \sin { }^{1} \varphi_{1}+{ }^{1} u_{3,1} \cos { }^{1} \varphi_{1} \cos { }^{1} \varphi_{2} \text {, } \\
& { }^{1} \kappa_{1}={ }^{1} \kappa_{\tau}={ }^{1} \varphi_{1,1} \cos { }^{1} \varphi_{2} \cos { }^{1} \varphi_{3}+{ }^{1} \varphi_{2,1} \sin { }^{1} \varphi_{3} \text {, } \\
& { }^{1} \kappa_{2}={ }^{1} \kappa_{n}={ }^{1} \varphi_{1,1} \cos { }^{1} \varphi_{2} \sin ^{1} \varphi_{3}-{ }^{1} \varphi_{2,1} \cos { }^{1} \varphi_{3} \text {, } \\
& { }^{1} \kappa_{3}={ }^{1} \kappa_{g}={ }^{1} \varphi_{1,1} \sin ^{1} \varphi_{2}+{ }^{1} \varphi_{3,1} \text {, } \\
& { }^{2} \varepsilon={ }^{2} e_{2}=-1-{ }^{2} u_{1,2}\left(\cos ^{2} \varphi_{3} \sin ^{2} \varphi_{1} \sin ^{2} \varphi_{2}+\cos ^{2} \varphi_{1} \sin ^{2} \varphi_{3}\right) \\
& +\left(1+{ }^{2} u_{2,2}\right) \cos ^{2} \varphi_{2} \cos ^{2} \varphi_{3} \\
& +{ }^{2} u_{3,2}\left(\sin ^{2} \varphi_{1} \sin ^{2} \varphi_{3}-\cos ^{2} \varphi_{1} \cos ^{2} \varphi_{3} \sin ^{2} \varphi_{2}\right), \\
& { }^{2} \gamma_{p}={ }^{2} e_{1}={ }^{2} u_{1,2}\left(\cos ^{2} \varphi_{1} \cos ^{2} \varphi_{3}-\sin ^{2} \varphi_{1} \sin ^{2} \varphi_{2} \sin ^{2} \varphi_{3}\right) \\
& +\left(1+{ }^{2} u_{2,2}\right) \cos ^{2} \varphi_{2} \sin ^{2} \varphi_{3} \\
& -{ }^{2} u_{3,2}\left(\cos ^{2} \varphi_{3} \sin ^{2} \varphi_{1}+\cos ^{2} \varphi_{1} \sin ^{2} \varphi_{2} \sin ^{2} \varphi_{3}\right) \text {, } \\
& { }^{2} \gamma_{n}={ }^{2} e_{3}={ }^{2} u_{1,2} \cos ^{2} \varphi_{2} \sin ^{2} \varphi_{1}+\left(1+{ }^{2} u_{2,2}\right) \sin ^{2} \varphi_{2}+{ }^{2} u_{3,2} \cos { }^{2} \varphi_{1} \cos ^{2} \varphi_{2} \text {, } \\
& { }^{2} \kappa_{1}={ }^{2} \kappa_{n}=-{ }^{2} \varphi_{1,2} \cos { }^{2} \varphi_{2} \sin ^{2} \varphi_{3}+{ }^{2} \varphi_{2,2} \cos ^{2} \varphi_{3} \text {, } \\
& { }^{2} \kappa_{2}={ }^{2} \kappa_{\tau}={ }^{2} \varphi_{1,2} \cos { }^{2} \varphi_{2} \cos { }^{2} \varphi_{3}+{ }^{2} \varphi_{2,2} \sin ^{2} \varphi_{3} \text {, } \\
& { }^{2} \kappa_{3}={ }^{2} \kappa_{g}={ }^{2} \varphi_{1,2} \sin ^{2} \varphi_{2}+{ }^{2} \varphi_{3,2} \text {, }
\end{aligned}
$$

where $(\cdot)_{, 1}=\frac{\partial}{\partial s_{1}}$ and $(\cdot)_{, 2}=\frac{\partial}{\partial s_{2}}$. 


\section{Kinematics of the pivots}

A typical pivot has been assumed to be a cylinder of radius $r$ and height $h$. The two bases of the pivot are assumed to be rigid bodies whose motion is described by a vector and an orthogonal tensor. The vector describes the displacement of a selected point (say $\boldsymbol{X}$ ) of the body and the tensor the rotation of the base (see Figure 4).

Henceforth, quantities related to each one of the two bases will be denoted by the indices $1 p$ and $2 p$, respectively.

Now, if

$$
{ }^{1 p} \boldsymbol{X}, \quad{ }^{2 p} \boldsymbol{X}
$$

are the selected points on the bases, we will denote by

$$
{ }^{1 p} \boldsymbol{u}\left({ }^{1 p} \boldsymbol{X}\right), \quad{ }^{2 p} \boldsymbol{u}\left({ }^{2 p} \boldsymbol{X}\right)
$$

the displacements of those points and by

$$
{ }^{1 p} \boldsymbol{R}, \quad{ }^{2 p} \boldsymbol{R}
$$

the rotations of the bases.

The deformation measures of the pivot are assumed as

$$
\begin{aligned}
{ }^{p} \boldsymbol{e} & ={ }^{1 p} \boldsymbol{R}^{\top}\left({ }^{2 p} \boldsymbol{u}-{ }^{1 p} \boldsymbol{u}\right)+\left({ }^{1 p} \boldsymbol{R}^{\top}-\boldsymbol{I}\right)\left({ }^{2 p} \boldsymbol{X}-{ }^{1 p} \boldsymbol{X}\right), \\
{ }^{p} \boldsymbol{E} & =\operatorname{skw}\left({ }^{1 p} \boldsymbol{R}^{\top}{ }^{2 p} \boldsymbol{R}\right)
\end{aligned}
$$

and can be proved to be frame-invariant or objective. The deformation measure related to ${ }^{p} \boldsymbol{E}$ is represented by its axial vector:

$$
{ }^{p} \boldsymbol{\kappa}={ }^{p} \boldsymbol{E}_{\times} .
$$

By considering the orthogonal tensor

$$
\boldsymbol{Q}=Q_{\alpha}{ }_{\beta} \boldsymbol{b}_{\alpha} \otimes \boldsymbol{b}_{\beta}
$$

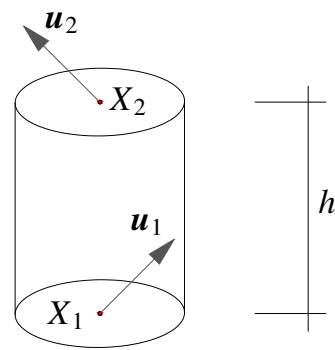

Figure 4. Pivot. 

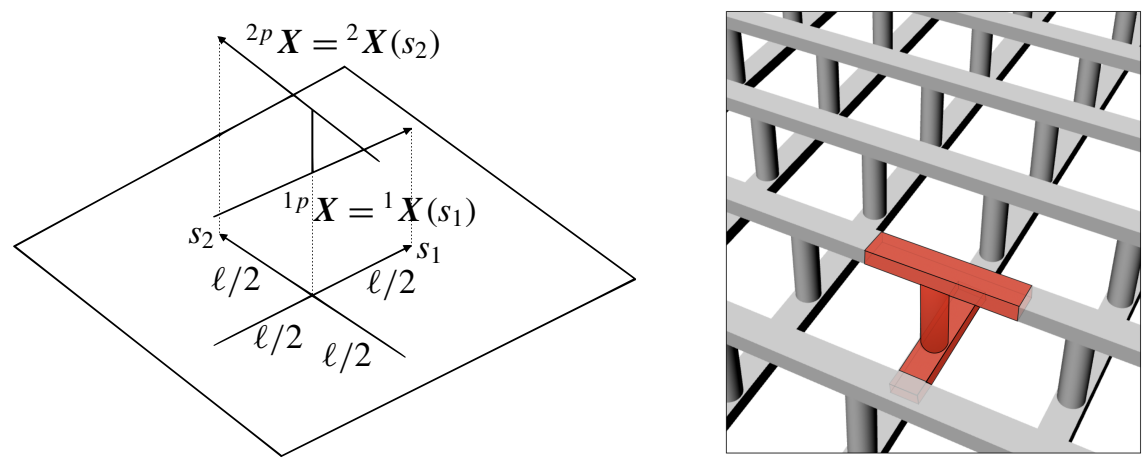

Figure 5. Unit cell.

which defines the basis transformation

$$
\boldsymbol{c}_{\alpha}=Q_{\beta \alpha} \boldsymbol{b}_{\beta}
$$

that in our case is

$$
\left(\begin{array}{l}
\boldsymbol{c}_{1} \\
\boldsymbol{c}_{2} \\
\boldsymbol{c}_{3}
\end{array}\right)=\left(\begin{array}{lll}
\boldsymbol{b}_{1} & \boldsymbol{b}_{2} & \boldsymbol{b}_{3}
\end{array}\right)\left(\begin{array}{rrr}
0 & -1 & 0 \\
1 & 0 & 0 \\
0 & 0 & 1
\end{array}\right),
$$

we obtain

$$
\begin{aligned}
{ }^{p} \boldsymbol{e} & =\left[{ }^{1 p} R_{j i}\left({ }^{2 p} u_{j}-{ }^{1 p} u_{j}\right)+\left({ }^{1 p} R_{j i}-\delta_{i j}\right)\left({ }^{2 p} X_{j}-{ }^{1 p} X_{j}\right)\right] \boldsymbol{b}_{i}, \\
{ }^{p} \boldsymbol{E} & =\operatorname{skw}\left({ }^{1 p} R_{\alpha i} Q_{\alpha \gamma}{ }^{2 p} \widetilde{R}_{\gamma \beta} Q_{j \beta} \boldsymbol{b}_{i} \otimes \boldsymbol{b}_{j}\right), \\
{ }^{p} \boldsymbol{\kappa} & ={ }^{p} E_{32} \boldsymbol{b}_{1}+{ }^{p} E_{13} \boldsymbol{b}_{2}+{ }^{p} E_{21} \boldsymbol{b}_{3} .
\end{aligned}
$$

\section{2D continuum kinematics}

The elementary cell is shown in Figure 5, where $\ell$ is the space between the two neighboring pivots. We make the identifications

$$
\begin{aligned}
{ }^{1 p} \boldsymbol{X} & ={ }^{1} \boldsymbol{X}\left(s_{1}\right), & { }^{2 p} \boldsymbol{X} & ={ }^{2} \boldsymbol{X}\left(s_{2}\right), \\
{ }^{1 p} \boldsymbol{u}\left({ }^{1 p} \boldsymbol{X}\right) & ={ }^{1} \boldsymbol{u}\left(s_{1}\right), & { }^{2 p} \boldsymbol{u}\left({ }^{2 p} \boldsymbol{X}\right) & ={ }^{2} \boldsymbol{u}\left(s_{2}\right), \\
{ }^{1 p} \boldsymbol{R} & ={ }^{1} \boldsymbol{R}\left(s_{1}\right), & { }^{2 p} \boldsymbol{R} & ={ }^{2} \boldsymbol{R}\left(s_{2}\right) .
\end{aligned}
$$

\section{2D strain energy}

The strain energy of a unit cell can be expressed as the sum of two contributions, specifically: 
(1) the term related to the fibers

$$
\begin{array}{r}
{ }^{f} \Pi_{c}=\frac{1}{2} \sum_{\alpha=1,2} \int_{-\ell / 2}^{\ell / 2}\left(\mathrm{E}^{f} A^{\alpha} \varepsilon^{2}+\mathrm{k}_{p} G^{f_{A}{ }^{\alpha} \gamma_{p}^{2}+\mathrm{k}_{n} G^{f} A^{\alpha} \gamma_{n}^{2}}\right. \\
\left.+\mathrm{q} G^{f} J_{\tau}{ }^{\alpha} \kappa_{\tau}^{2}+\mathrm{E} J_{n}{ }^{\alpha} \kappa_{n}^{2}+\mathrm{E} J_{g}{ }^{\alpha} \kappa_{g}^{2}\right) \mathrm{d} s_{\alpha}
\end{array}
$$

where $\mathrm{E}$ and $G$ are the Young and shear moduli, respectively, ${ }^{f} A$ is the crosssection area, $J_{g}$ is the moment of geodesic flexural inertia, $J_{n}$ is the moment of normal flexural inertia, ${ }^{f} J_{\tau}$ is the polar moment inertia, and $\mathrm{k}_{p}, \mathrm{k}_{n}$, and $q$ are positive constants,

(2) the term related to the pivot connecting the fibers

${ }^{p} \Pi_{c}=\frac{1}{2 h}\left(\mathrm{E}^{p} A^{p} e_{3}{ }^{2}+4 \mathrm{E}^{p} J\left({ }^{p} \kappa_{1}{ }^{2}+{ }^{p} \kappa_{2}{ }^{2}\right)+G^{p} J_{\tau}{ }^{p} \kappa_{3}{ }^{2}+\frac{12 \mathrm{E}^{p} J}{h^{2}}\left({ }^{p} e_{1}{ }^{2}+{ }^{p} e_{2}{ }^{2}\right)\right)$

where ${ }^{p} A,{ }^{p} J_{\tau}$, and ${ }^{p} J$ are the area and the torsional and flexural inertial moments of the cross-area of the pivot, respectively.

The density of the strain energy of the two-dimensional elastic surface is defined as the average strain energy per unit cell, i.e.,

$$
\pi=\pi_{f}+\pi_{p}=\frac{1}{{ }^{c} A}\left({ }^{f} \Pi_{c}+{ }^{p} \Pi_{c}\right)
$$

where ${ }^{c} A$ is the two-dimensional measure of the unit cell, i.e., $\ell^{2}$. This makes explicit the contributions of the fibers $\pi_{f}$ and of the pivots $\pi_{p}$. Having in mind an asymptotic homogenization procedure, we can imagine an infinite distribution of fibers on the area $\ell^{2}$ along both the original directions of the discrete net of fibers and express the homogenized strain energy as

$$
\begin{aligned}
\pi_{f}=\frac{1}{\ell^{2}} \sum_{\alpha=1,2} \frac{\ell}{2}\left(\mathrm{E}^{f} A^{\alpha} \varepsilon^{2}+\mathrm{k}_{p} G^{f} A^{\alpha} \gamma_{p}^{2}+\mathrm{k}_{n} G^{f} A^{\alpha} \gamma_{n}^{2}\right. \\
\left.+\mathrm{q} G^{f} J_{\tau}{ }^{\alpha} \kappa_{\tau}^{2}+\mathrm{E} J_{n}{ }^{\alpha} \kappa_{n}^{2}+\mathrm{E} J_{g}{ }^{\alpha} \kappa_{g}^{2}\right)
\end{aligned}
$$

and

$$
\pi_{p}=\frac{1}{2 \ell^{2} h}\left(\mathrm{E}^{p} A^{p} e_{3}{ }^{2}+4 \mathrm{E}^{p} J\left({ }^{p} \kappa_{1}{ }^{2}+{ }^{p} \kappa_{2}{ }^{2}\right)+G^{p} J_{\tau}{ }^{p} \kappa_{3}{ }^{2}+\frac{12 \mathrm{E}^{p} J}{h^{2}}\left({ }^{p} e_{1}{ }^{2}+{ }^{p} e_{2}{ }^{2}\right)\right) .
$$

As a result, we have for the strain energy density

$$
\begin{gathered}
\pi_{f}=\frac{1}{2}\left[{ }^{f} \mathscr{K}_{e}\left({ }^{1} \varepsilon^{2}+{ }^{2} \varepsilon^{2}\right)+\mathscr{K}_{\gamma_{p}}\left({ }^{1} \gamma_{p}{ }^{2}+{ }^{2} \gamma_{p}^{2}\right)+\mathscr{K}_{\gamma_{n}}\left({ }^{1} \gamma_{n}{ }^{2}+{ }^{2} \gamma_{n}{ }^{2}\right)\right. \\
\left.+{ }^{f} \mathscr{K}_{\tau}\left({ }^{1} \kappa_{\tau}{ }^{2}+{ }^{2} \kappa_{\tau}{ }^{2}\right)+\mathscr{K}_{n}\left({ }^{1} \kappa_{n}{ }^{2}+{ }^{2} \kappa_{n}{ }^{2}\right)+\mathscr{K}_{g}\left({ }^{1} \kappa_{g}{ }^{2}+{ }^{2} \kappa_{g}{ }^{2}\right)\right], \\
\pi_{p}=\frac{1}{2}\left[\mathscr{K}_{b}\left({ }^{p} \kappa_{1}{ }^{2}+{ }^{p} \kappa_{2}{ }^{2}\right)+{ }^{p} \mathscr{K}_{\tau}{ }^{p} \kappa_{3}{ }^{2}+\mathscr{K}_{\gamma}\left({ }^{p} e_{1}{ }^{2}+{ }^{p} e_{2}{ }^{2}\right)+{ }^{p} \mathscr{K}_{e}{ }^{p} e_{3}{ }^{2}\right]
\end{gathered}
$$


where ${ }^{f} \mathscr{K}_{e}, \mathscr{K}_{\gamma_{p}}, \mathscr{K}_{\gamma_{n}},{ }^{f} \mathscr{K}_{\tau}, \mathscr{K}_{n}$, and $\mathscr{K}_{g}$ are positive constitutive parameters, assumed equal for the two continuous distributions of embedded beams, and representing the stiffnesses related to the elongation, shear, twisting, normal, and geodesic bending, respectively, while ${ }^{p_{\mathscr{K}}}{ }_{{ }}{ }_{\mathscr{K}_{\tau}}, \mathscr{K}_{\gamma}$, and $\mathscr{K}_{b}$ are positive constitutive parameters related to the elongation, torsion, shear, and bending stiffnesses of the pivots. In particular, we have

$$
\begin{aligned}
f_{\mathscr{K}_{e}} & =\frac{\mathrm{E}^{f} A}{\ell}, \mathscr{K}_{\gamma_{p}}=\frac{\mathrm{k}_{p} G^{f} A}{\ell}, \mathscr{K}_{\gamma_{n}}=\frac{\mathrm{k}_{n} G^{f} A}{\ell},{ }^{f} \mathscr{K}_{\tau}=\frac{\mathrm{q} G^{f} J_{\tau}}{\ell}, \mathscr{K}_{n}=\frac{\mathrm{E} J_{n}}{\ell}, \\
\mathscr{K}_{g} & =\frac{\mathrm{E} J_{g}}{\ell}, \quad p_{\mathscr{K}_{e}}=\frac{\mathrm{E}^{p} A}{\ell^{2} h}, \quad p_{\mathscr{K}_{\tau}}=\frac{G^{p} J_{\tau}}{\ell^{2} h}, \quad \mathscr{K}_{\gamma}=\frac{12 \mathrm{E}^{p} J}{\ell^{2} h^{3}}, \mathscr{K}_{b}=\frac{4 \mathrm{E}^{p} J}{\ell^{2} h} .
\end{aligned}
$$

\section{Numerical simulations and discussion}

We test the proposed model numerically to assess its predictive potential. To this end we use the principle of virtual work in the form

$$
-\delta \int_{\mathscr{B}}\left(\pi_{f}+\pi_{p}\right) \mathrm{d} \Omega+\delta \mathscr{W}^{\text {ext }}=0
$$

where $\delta \mathscr{W}^{\text {ext }}$ is the virtual external work done by the external actions acting on the displacements ${ }^{\alpha} u_{i}$ and the angles ${ }^{\alpha} \varphi_{i}$, and the integration is performed on the rectangular two-dimensional region $\mathscr{B}$. In particular, we compare the model (30) with a refined three-dimensional simulation based on a Cauchy-continuum model with a highly detailed description of the geometry of fibers and connections and a previous version of a reduced model presented in [Giorgio et al. 2017]. The size of the sample is $7 \times 21 \mathrm{~cm}$, and the fibers have a rectangular cross-section of height $1 \mathrm{~mm}$ and width $1.6 \mathrm{~mm}$. The fibers constrained at the short side are 10 in number for each direction, and therefore, their pitch is $4.95 \mathrm{~mm}$. The connections are cylinders with diameter $0.9 \mathrm{~mm}$. The considered material is a polyamide characterized by Young's modulus $1600 \mathrm{MPa}$ and Poisson's ratio 0.4. To compare these models, we numerically perform a bias test for three kinds of pantographic sheet, changing the height of the cylindrical connections. Specifically, we compare the results for pivots whose heights are $1 \mathrm{~mm}, 2.5 \mathrm{~mm}$, and $5 \mathrm{~mm}$. Of course, the deformability of the connection is largely affected by the height of the pivots, and hence, the hypotheses made in the present paper are expected to be more appropriate as the height of these connections increases. Figure 6 shows the plots of the longitudinal reactions obtained in the bias extension test by imposing a displacement in the direction of the length of the sample for different heights of the connections and for the three models considered: (1) the detailed Cauchy model, (2) the latent internal microstructure two-dimensional model [Giorgio et al. 2017] in which the sole kinematical mode of pivot deformation is torsion, and (3) the present twodimensional model with enhanced deformation capabilities. As expected, the new 


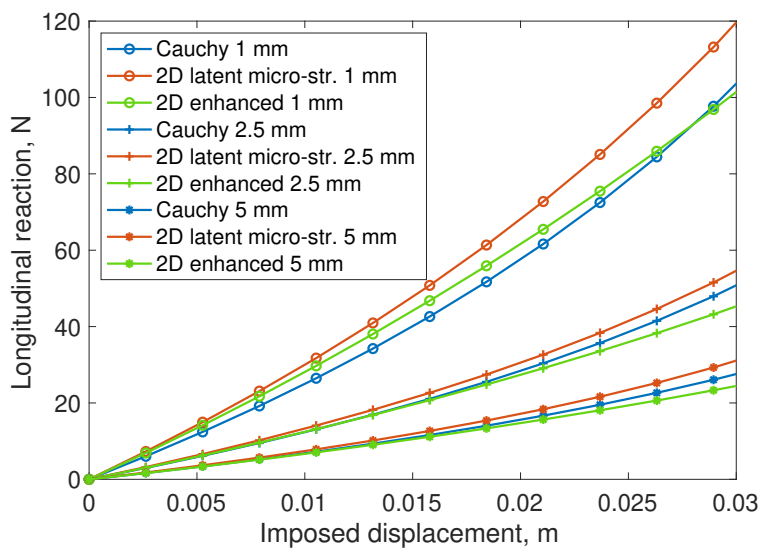

Figure 6. Reaction versus displacements using different models.
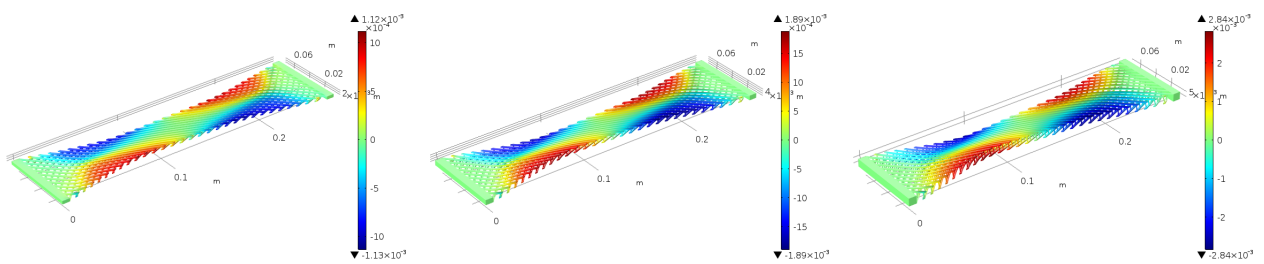

Figure 7. Cauchy 3D model with pivot heights $1 \mathrm{~mm}, 2.5 \mathrm{~mm}$, and $5 \mathrm{~mm}$. The colors show the transversal displacement.
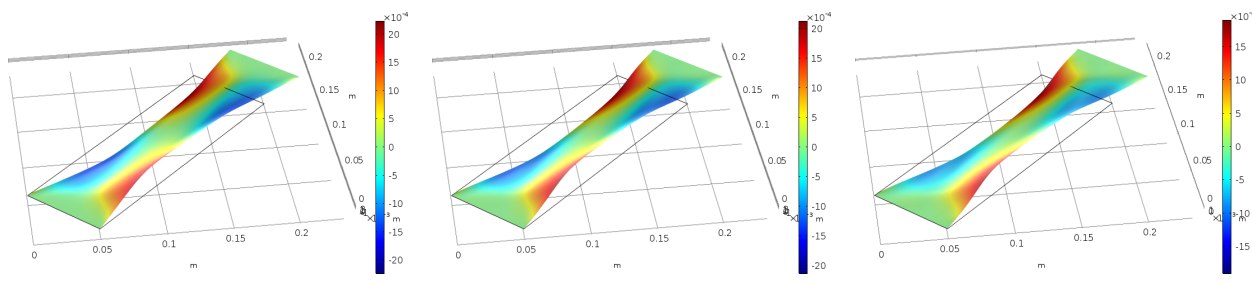

Figure 8. Reduced 2D model with pivot heights $1 \mathrm{~mm}, 2.5 \mathrm{~mm}$, and $5 \mathrm{~mm}$. The colors show the transversal displacement.

model performs better as the height of the connections increases due to their greater induced deformation.

Figure 7 depicts a chiral effect due to the particular arrangement of the microstructure. Indeed, the transverse displacement, highlighted in color, clearly reveals an overall torsion of the entire sample in the extension test. The same behavior is obtained by using the present model, as seen in Figure 8. We remark that the same effect cannot be obtained with the previously developed model [Giorgio 

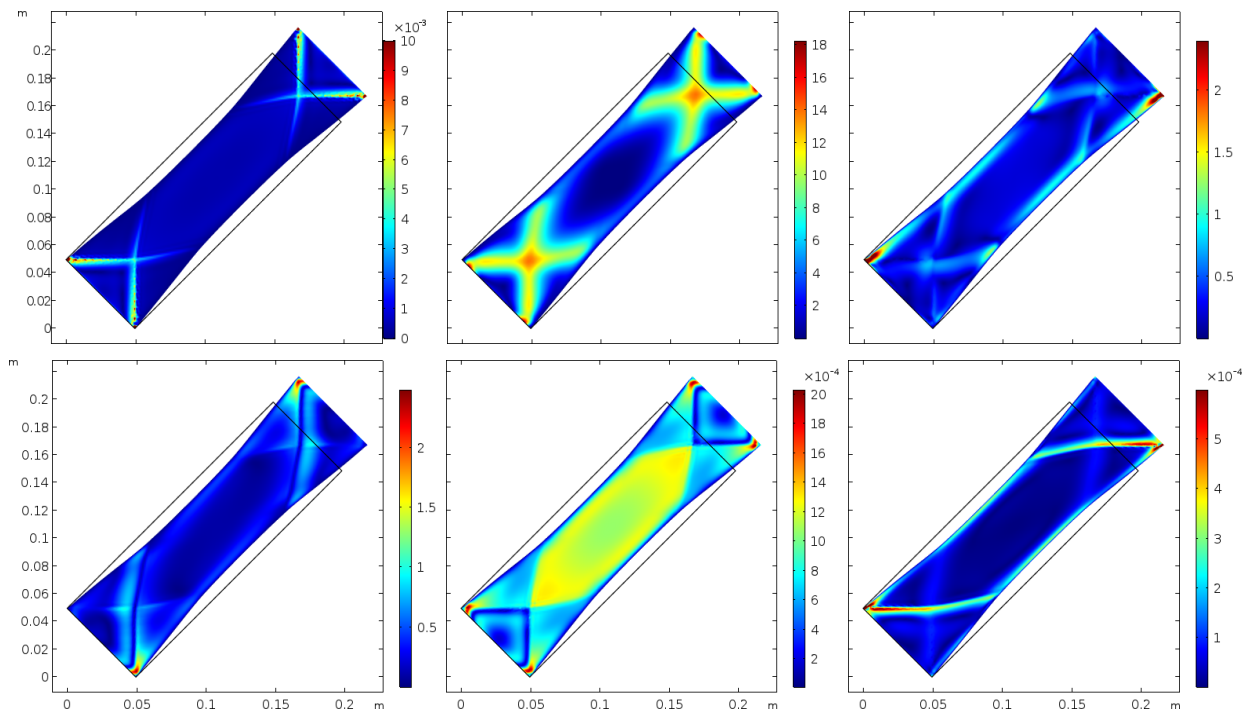

Figure 9. Reduced 2D model for height pivot $5 \mathrm{~mm}$ with an imposed displacement of $2.63 \mathrm{~cm}$ : deformation measures of fibers (from left to right and top to bottom) elongation, geodesic bending, out-of-plane bending, twisting, tangent plane shear, and out-ofplane shear.
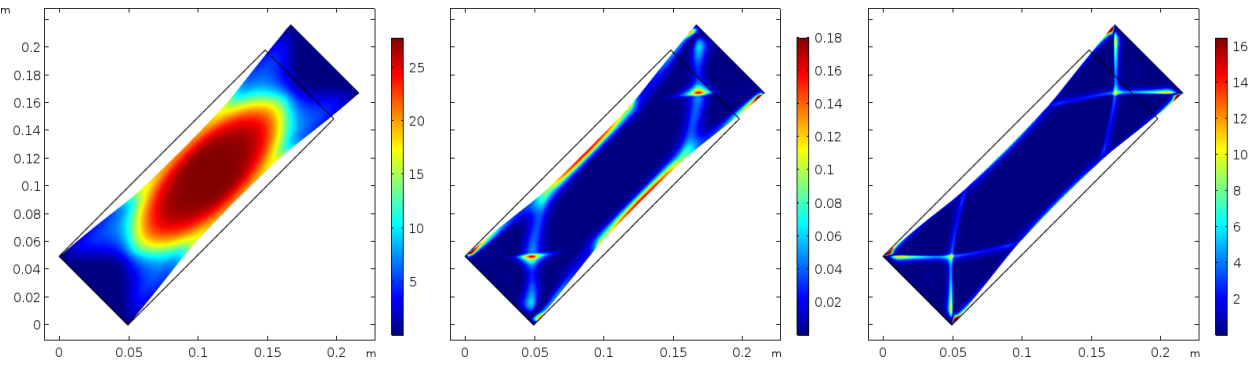

Figure 10. Reduced 2D model for height pivot $5 \mathrm{~mm}$ with an imposed displacement of $2.63 \mathrm{~cm}$ : deformation measures of pivots (from left to right) twisting, bending, and tangent plane shear. The elongation of the pivot is negligible.

et al. 2017], which does not take into account the offset of fibers and the complex deformations of the connections.

The asymmetry due to the microstructure in an extension test involving symmetric boundary conditions is also evident in the considered deformation measures, as shown in Figures 9 and 10. 

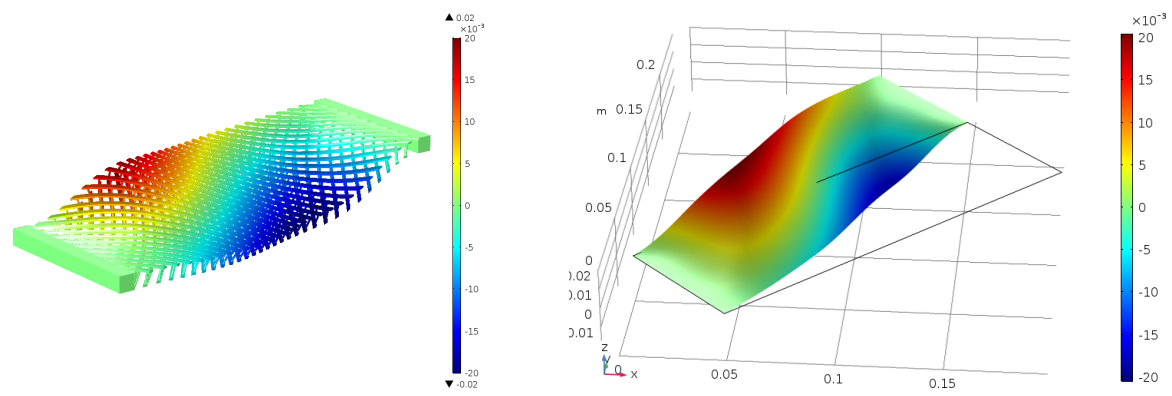

Figure 11. Buckled shapes in a shear test: Cauchy model (left) and 2D enhanced model (right).

Finally, we note that using the present 2D enhanced model, it is possible to predict the typical buckling behavior of the pantographic sheet as shown in Figure 11.

\section{Conclusions}

The comparison of the numerical results obtained by using the refined 3D model and the present reduced order model is very suggestive. In the reduced order model, it has been possible to introduce the initial and actual dimensions of the interconnecting pivots. Therefore, pivot deformation and the associated deformation energy are meaningful in both the refined and the reduced order models. Consequently a comparison of their predictive capabilities is straightforward.

The micro-macro identification which we have presented is based on the structure of the reduced order model and a fit of macroconstitutive parameters assuming the microconstitutive parameters and the predictions of the micromodel to be known.

Several research perspectives involving mathematical, numerical, and mechanical issues are opened by the present model, including the convergence of the micromodel to the macro multifield reduced order model.

We expect that the methods presented in [Pideri and Seppecher 1997; Alibert and Della Corte 2019; Abdoul-Anziz and Seppecher 2018] can be adapted to address this issue in the present context.

The issue of appropriate mechanical modeling for systems of the kind considered here presents interesting challenges that we intend to explore in future investigations. In this regard the present work may be considered a prototypical problem in the more general context of mathematical modeling of metamaterials.

Finally, it remains to investigate further optimization of the numerical strategies, the numerical codes, and the computational techniques to be used in the study of metamaterials. 
Both structural optimization and metamaterial design require the iterative solution of many deformation problems. To be able to use very efficient numerical integration schemes, specially tailored for strongly nonlinear and potentially unstable systems, is of great importance. Relevant developments are presented in [Cazzani et al. 2016; Turco et al. 2016a; 2019; 2018; Greco et al. 2019; Khakalo and Niiranen 2017; Maurin et al. 2019].

\section{Acknowledgement}

This research has been funded by the Italian Ministero dell'Istruzione dell'Università e della Ricerca (MIUR) through the PRIN 2015.

\section{References}

[Abdoul-Anziz and Seppecher 2018] H. Abdoul-Anziz and P. Seppecher, "Strain gradient and generalized continua obtained by homogenizing frame lattices", Math. Mech. Complex Syst. 6:3 (2018), 213-250.

[Alibert and Della Corte 2019] J.-J. Alibert and A. Della Corte, "Homogenization of nonlinear inextensible pantographic structures by $\Gamma$-convergence", Math. Mech. Complex Syst. 7:1 (2019), 1-24.

[Alibert et al. 2003] J.-J. Alibert, P. Seppecher, and F. Dell' Isola, “Truss modular beams with deformation energy depending on higher displacement gradients", Math. Mech. Solids 8:1 (2003), $51-73$.

[Andreaus et al. 2018] U. Andreaus, M. Spagnuolo, T. Lekszycki, and S. R. Eugster, "A Ritz approach for the static analysis of planar pantographic structures modeled with nonlinear EulerBernoulli beams", Contin. Mech. Thermodyn. 30:5 (2018), 1103-1123.

[Barchiesi and Placidi 2017] E. Barchiesi and L. Placidi, "A review on models for the 3D statics and 2D dynamics of pantographic fabrics", pp. 239-258 in Wave dynamics and composite mechanics for microstructured materials and metamaterials, edited by M. A. Sumbatyan, Adv. Struct. Mater. 59, Springer, 2017.

[Barchiesi et al. 2019a] E. Barchiesi, G. Ganzosch, C. Liebold, L. Placidi, R. Grygoruk, and W. H. Müller, "Out-of-plane buckling of pantographic fabrics in displacement-controlled shear tests: experimental results and model validation", Contin. Mech. Thermodyn. 31:1 (2019), 33-45.

[Barchiesi et al. 2019b] E. Barchiesi, M. Spagnuolo, and L. Placidi, "Mechanical metamaterials: a state of the art", Math. Mech. Solids 24:1 (2019), 212-234.

[Cazzani et al. 2016] A. Cazzani, M. Malagù, and E. Turco, "Isogeometric analysis of plane-curved beams", Math. Mech. Solids 21:5 (2016), 562-577.

[Cazzani et al. 2018a] A. Cazzani, N. L. Rizzi, F. Stochino, and E. Turco, "Modal analysis of laminates by a mixed assumed-strain finite element model”, Math. Mech. Solids 23:1 (2018), 99-119.

[Cazzani et al. 2018b] A. Cazzani, M. Serra, F. Stochino, and E. Turco, "A refined assumed strain finite element model for statics and dynamics of laminated plates", Contin. Mech. Thermodyn. (online publication September 2018).

[De Angelo et al. 2019a] M. De Angelo, E. Barchiesi, I. Giorgio, and B. E. Abali, "Numerical identification of constitutive parameters in reduced-order bi-dimensional models for pantographic structures: application to out-of-plane buckling", Arch. Appl. Mech. 89:7 (2019), 1333-1358. 
[De Angelo et al. 2019b] M. De Angelo, M. Spagnuolo, F. D’Annibale, A. Pfaff, K. Hoschke, A. Misra, C. Dupuy, P. Peyre, J. Dirrenberger, and M. Pawlikowski, "The macroscopic behavior of pantographic sheets depends mainly on their microstructure: experimental evidence and qualitative analysis of damage in metallic specimens", Contin. Mech. Thermodyn. 31:4 (2019), 1181-1203.

[dell'Isola et al. 2015] F. dell'Isola, T. Lekszycki, M. Pawlikowski, R. Grygoruk, and L. Greco, "Designing a light fabric metamaterial being highly macroscopically tough under directional extension: first experimental evidence", Z. Angew. Math. Phys. 66:6 (2015), 3473-3498.

[dell'Isola et al. 2019] F. dell'Isola, P. Seppecher, J. J. Alibert, and et al., "Pantographic metamaterials: an example of mathematically driven design and of its technological challenges", Contin. Mech. Thermodyn. 31:4 (2019), 851-884.

[Eremeyev 2018] V. A. Eremeyev, “A nonlinear model of a mesh shell”, Mech. Solids 53:4 (2018), 464-469.

[Eremeyev 2019] V. A. Eremeyev, "Two- and three-dimensional elastic networks with rigid junctions: modeling within the theory of micropolar shells and solids", Acta Mech. 230:11 (2019), 3875-3887.

[Eremeyev and Pietraszkiewicz 2006] V. A. Eremeyev and W. Pietraszkiewicz, "Local symmetry group in the general theory of elastic shells", J. Elasticity 85:2 (2006), 125-152.

[Eremeyev and Pietraszkiewicz 2016] V. A. Eremeyev and W. Pietraszkiewicz, "Material symmetry group and constitutive equations of micropolar anisotropic elastic solids", Math. Mech. Solids 21:2 (2016), 210-221.

[Eremeyev et al. 2018] V. A. Eremeyev, F. dell'Isola, C. Boutin, and D. Steigmann, "Linear pantographic sheets: existence and uniqueness of weak solutions", J. Elasticity 132:2 (2018), 175-196.

[Eugster et al. 2019] S. R. Eugster, F. dell'Isola, and D. J. Steigmann, "Continuum theory for mechanical metamaterials with a cubic lattice substructure”, Math. Mech. Complex Syst. 7:1 (2019), $75-98$.

[Giorgio 2016] I. Giorgio, "Numerical identification procedure between a micro-Cauchy model and a macro-second gradient model for planar pantographic structures", Z. Angew. Math. Phys. 67:4 (2016), art. id. 95.

[Giorgio et al. 2017] I. Giorgio, N. L. Rizzi, and E. Turco, "Continuum modelling of pantographic sheets for out-of-plane bifurcation and vibrational analysis", Proc. A. 473:2207 (2017), art. id. 20170636.

[Greco et al. 2019] L. Greco, M. Cuomo, and L. Contrafatto, "A quadrilateral $G^{1}$-conforming finite element for the Kirchhoff plate model", Comput. Methods Appl. Mech. Engrg. 346 (2019), 913-951.

[Khakalo and Niiranen 2017] S. Khakalo and J. Niiranen, "Isogeometric analysis of higher-order gradient elasticity by user elements of a commercial finite element software", Comput.-Aided Des. 82 (2017), 154-169.

[Maurin et al. 2019] F. Maurin, F. Greco, and W. Desmet, "Isogeometric analysis for nonlinear planar pantographic lattice: discrete and continuum models", Contin. Mech. Thermodyn. 31:4 (2019), 1051-1064.

[Pideri and Seppecher 1997] C. Pideri and P. Seppecher, "A second gradient material resulting from the homogenization of an heterogeneous linear elastic medium", Contin. Mech. Thermodyn. 9:5 (1997), 241-257.

[Placidi et al. 2016] L. Placidi, E. Barchiesi, E. Turco, and N. L. Rizzi, "A review on 2D models for the description of pantographic fabrics", Z. Angew. Math. Phys. 67:5 (2016), art. id. 121.

[Seppecher et al. 2011] P. Seppecher, J.-J. Alibert, and F. dell'Isola, "Linear elastic trusses leading to continua with exotic mechanical interactions”, J. Phys. Conf. Ser. 319:1 (2011), art. id. 012018. 
[Spagnuolo et al. 2017] M. Spagnuolo, K. Barcz, A. Pfaff, F. dell'Isola, and P. Franciosi, "Qualitative pivot damage analysis in aluminum printed pantographic sheets: numerics and experiments", Mech. Res. Commun. 83 (2017), 47-52.

[Steigmann and dell'Isola 2015] D. J. Steigmann and F. dell'Isola, "Mechanical response of fabric sheets to three-dimensional bending, twisting, and stretching", Acta Mech. Sin. 31:3 (2015), 373382.

[Turco et al. 2016a] E. Turco, F. dell'Isola, A. Cazzani, and N. L. Rizzi, "Hencky-type discrete model for pantographic structures: numerical comparison with second gradient continuum models", Z. Angew. Math. Phys. 67:4 (2016), art. id. 85.

[Turco et al. 2016b] E. Turco, F. dell'Isola, N. L. Rizzi, R. Grygoruk, W. H. Müller, and C. Liebold, "Fiber rupture in sheared planar pantographic sheets: numerical and experimental evidence", Mech. Res. Commun. 76 (2016), 86-90.

[Turco et al. 2018] E. Turco, A. Misra, M. Pawlikowski, F. dell'Isola, and F. Hild, "Enhanced PiolaHencky discrete models for pantographic sheets with pivots without deformation energy: numerics and experiments", Int. J. Solids. Struct. 147 (2018), 94-109.

[Turco et al. 2019] E. Turco, A. Misra, R. Sarikaya, and T. Lekszycki, "Quantitative analysis of deformation mechanisms in pantographic substructures: experiments and modeling", Contin. Mech. Thermodyn. 31:1 (2019), 209-223.

Received 23 Jul 2019. Accepted 11 Nov 2019.

IVAN GIORGIO: ivan.giorgio@uniroma1.it

International Research Center on Mathematics and Mechanics of Complex Systems,

Università degli studi dell' Aquila, L'Aquila, Italy

NiCOLA L. RIZZI: nicolaluigi.rizzi@uniroma3.it

Department of Architecture, Università degli studi Roma Tre, Roma, Italy

UGO ANDREAUS: ugo.andreaus@uniroma1.it

Department of Structural and Geotechnical Engineering, Università degli studi di Roma "La

Sapienza", Roma, Italy

DAVID J. STEIGMAnN: dsteigmann@berkeley .edu

Department of Mechanical Engineering, University of California, Berkeley, Berkeley, CA, United States 



\section{Guidelines for Authors}

Authors may submit manuscripts in PDF format on-line at the submission page.

Originality. Submission of a manuscript acknowledges that the manuscript is original and and is not, in whole or in part, published or under consideration for publication elsewhere. It is understood also that the manuscript will not be submitted elsewhere while under consideration for publication in this journal.

Language. Articles in MEMOCS are usually in English, but articles written in other languages are welcome.

Required items. A brief abstract of about 150 words or less must be included. It should be selfcontained and not make any reference to the bibliography. If the article is not in English, two versions of the abstract must be included, one in the language of the article and one in English. Also required are keywords and a Mathematics Subject Classification or a Physics and Astronomy Classification Scheme code for the article, and, for each author, postal address, affiliation (if appropriate), and email address if available. A home-page URL is optional.

Format. Authors are encouraged to use $\mathrm{IAT}_{\mathrm{E} X}$ and the standard amsart class, but submissions in other varieties of $\mathrm{T}_{\mathrm{E}} \mathrm{X}$, and exceptionally in other formats, are acceptable. Initial uploads should normally be in PDF format; after the refereeing process we will ask you to submit all source material.

References. Bibliographical references should be complete, including article titles and page ranges. All references in the bibliography should be cited in the text. The use of $\mathrm{BIBT}_{\mathrm{E}} \mathrm{X}$ is preferred but not required. Tags will be converted to the house format, however, for submission you may use the format of your choice. Links will be provided to all literature with known web locations and authors are encouraged to provide their own links in addition to those supplied in the editorial process.

Figures. Figures must be of publication quality. After acceptance, you will need to submit the original source files in vector graphics format for all diagrams in your manuscript: vector EPS or vector PDF files are the most useful.

Most drawing and graphing packages - Mathematica, Adobe Illustrator, Corel Draw, MATLAB, etc. - allow the user to save files in one of these formats. Make sure that what you are saving is vector graphics and not a bitmap. If you need help, please write to graphics@msp.org with as many details as you can about how your graphics were generated.

Bundle your figure files into a single archive (using zip, tar, rar or other format of your choice) and upload on the link you been provided at acceptance time. Each figure should be captioned and numbered so that it can float. Small figures occupying no more than three lines of vertical space can be kept in the text ("the curve looks like this:"). It is acceptable to submit a manuscript with all figures at the end, if their placement is specified in the text by means of comments such as "Place Figure 1 here". The same considerations apply to tables.

White Space. Forced line breaks or page breaks should not be inserted in the document. There is no point in your trying to optimize line and page breaks in the original manuscript. The manuscript will be reformatted to use the journal's preferred fonts and layout.

Proofs. Page proofs will be made available to authors (or to the designated corresponding author) at a Web site in PDF format. Failure to acknowledge the receipt of proofs or to return corrections within the requested deadline may cause publication to be postponed. 
Mathematics and Mechanics of Complex Systems vol. 7 no. 4

Equilibrium paths of Hencky pantographic beams in a three-point bending problem

Emilio Turco and Emilio Barchiesi

A two-dimensional continuum model of pantographic sheets moving in a 3D space and accounting for the offset and relative rotations of the fibers

Ivan Giorgio, Nicola L. Rizzi, Ugo Andreaus and David J. Steigmann

MEMOCS is a journal of the International Research Center for the Mathematics and Mechanics of Complex Systems at the Università dell' Aquila, Italy.

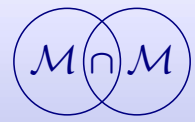

